\title{
Nonlinear Force-Free Modeling of Coronal Magnetic Fields. II. Modeling a Filament Arcade and Simulated Chromospheric and Photospheric Vector Fields
}

\author{
Thomas R. Metcalf • Marc L. DeRosa • Carolus J. Schrijver • Graham Barnes • \\ Adriaan A. van Ballegooijen • Thomas Wiegelmann • Michael S. Wheatland • \\ Gherardo Valori · James M. McTtiernan
}

Received: 3 April 2007 / Accepted: 11 December 2007 / Published online: 1 February 2008 (C) Springer Science+Business Media B.V. 2008

\begin{abstract}
We compare a variety of nonlinear force-free field (NLFFF) extrapolation algorithms, including optimization, magneto-frictional, and Grad-Rubin-like codes, applied to a solar-like reference model. The model used to test the algorithms includes realistic photospheric Lorentz forces and a complex field including a weakly twisted, right helical flux bundle. The codes were applied to both forced "photospheric" and more force-free "chromospheric" vector magnetic field boundary data derived from the model. When applied to the chromospheric boundary data, the codes are able to recover the presence of the flux bun-
\end{abstract}

On 07/07/2007, the NLFFF team was saddened by the news that Tom Metcalf had died as the result of an accident. We remain grateful for having had the opportunity to benefit from his unwavering dedication to the problems encountered in attempting to understand the Sun's magnetic field; Tom had completed this paper several months before his death, leading the team through the many steps described above.

T.R. Metcalf · G. Barnes

Northwest Research Associates, Colorado Research Associates Division, 3380 Mitchell Ln., Boulder, CO 80301, USA

M.L. DeRosa · C.J. Schrijver ( $₫)$

Lockheed Martin Advanced Technology Center, Dept. ADBS, Bldg. 252, 3251 Hanover St., Palo Alto, CA 94304, USA

e-mail: schryver@Imsal.com

A.A. van Ballegooijen

Smithsonian Astrophysical Observatory, 60 Garden St., Cambridge, MA 02138, USA

T. Wiegelmann

Max Planck Institut für Sonnensystemforschung, Katlenburg-Lindau, Germany

M.S. Wheatland

School of Physics, University of Sydney, Sydney, NSW 2006, Australia

G. Valori

Astrophysical Institute Potsdam, 14482 Potsdam, Germany

J.M. McTtiernan

Space Sciences Laboratory, University of California, Berkeley, CA 94720, USA 
dle and the field's free energy, though some details of the field connectivity are lost. When the codes are applied to the forced photospheric boundary data, the reference model field is not well recovered, indicating that the combination of Lorentz forces and small spatial scale structure at the photosphere severely impact the extrapolation of the field. Preprocessing of the forced photospheric boundary does improve the extrapolations considerably for the layers above the chromosphere, but the extrapolations are sensitive to the details of the numerical codes and neither the field connectivity nor the free magnetic energy in the full volume are well recovered. The magnetic virial theorem gives a rapid measure of the total magnetic energy without extrapolation though, like the NLFFF codes, it is sensitive to the Lorentz forces in the coronal volume. Both the magnetic virial theorem and the Wiegelmann extrapolation, when applied to the preprocessed photospheric boundary, give a magnetic energy which is nearly equivalent to the value derived from the chromospheric boundary, but both underestimate the free energy above the photosphere by at least a factor of two. We discuss the interpretation of the preprocessed field in this context. When applying the NLFFF codes to solar data, the problems associated with Lorentz forces present in the low solar atmosphere must be recognized: the various codes will not necessarily converge to the correct, or even the same, solution.

Keywords Nonlinear force-free field modeling · Solar magnetic field · Coronal magnetic field · Methods: numerical

\section{Introduction}

The magnetic field that threads the photosphere shapes the Sun's entire atmosphere, and provides the power for its heating and frequent explosions. The study of coronal loops, coronal heating, and solar activity would, therefore, greatly benefit from knowledge of the embedding field geometry. The study of flares and other eruptive phenomena requires that we understand where and how free energy resides within the field and how these phenomena depend on the field topology. There is therefore an urgent need within the solar physics community for methods to both model the coronal field and quantify the success of such methods.

The simplest way to model the coronal field is to assume that it is potential, i.e. that it carries no electric current. This assumption has proven to be adequate for many quiescent, old active regions and even for the noneruptive global coronal-heliospheric interface (e.g., Wang and Sheeley, 1990; Hoeksema, 1991; Schrijver and DeRosa, 2003). The assumption fails, of course, in many interesting cases because it is by definition wrong when we look at regions that can support flares, filament eruptions, or coronal mass ejections: such regions contain free energy in the form of strong electrical currents.

Whereas full MHD modeling of the coronal field is desirable, the routine application of such models to the study of many active regions, or to the long-term evolution of large volumes of the solar corona, is not yet computationally feasible; MHD models are far too time consuming for that. A convenient approximation that can accommodate electrical currents is the assumption that Lorentz forces are negligible, i.e., that the field evolves slowly through a series of quasi-equilibrium states. This approximation applies for any slowly evolving low- $\beta$ plasma where the gas pressure gradients and the gravitational forces are much smaller than the magnetic pressure and curvature forces and, in particular, is expected to apply above solar active regions. 
This force-free field approximation implies that electrical currents run only along the field lines. If the ratio of field strength to current density is constant throughout a volume, the approximation is referred to as a linear force-free field (LFFF). This is a rather restrictive assumption, but it enables fast and straightforward modeling (e.g., Gary, 1989). Here, we focus on the more general approximation that the ratio of field strength and current density can vary from field line to field line, giving us the nonlinear force-free field (NLFFF).

NLFFF modeling is more demanding of computational resources than the potential or LFFF case (but still tractable for routine studies, unlike MHD), and is sensitive - as is all nonlinear modeling - to the initial and boundary conditions. In the first of this series of papers, Schrijver et al. (2006) (hereafter referred to as Paper I) compared the results of model computations using six different NLFFF algorithms, including optimization, magneto-frictional, Grad - Rubin-based and Green's-function-based methods. They showed that NLFFF models can be successful, provided that all side boundary conditions are applied, in combination with a judiciously chosen initial condition.

The comparison of the various methods in Paper I was based on analytically known NLFFFs from Low and Lou (1990). These fields have small gradients in the ratio $\alpha$ of field to current, and patterns of current and magnetic field that are not particularly reminiscent of the real Sun. Here, we continue that exercise by applying the methods to a much more realistic reference field. As described in Section 2.1, the reference field is a modified solar observation to which a current system was added to simulate a filament arcade. This combined system was then relaxed to a force-free state using a magneto-frictional code. The lower boundary vector field was supplied with information on the side boundaries (see below for details), as in our earlier study, to the NLFFF modelers without revealing the properties of the reference field above the lower boundary.

The present study enables us to make several important advances relative to the work in Paper I that go beyond the obvious difference of using a more solar-like test field. Foremost among these is our attempt to mimic the analysis of real observational data while still being in the position of knowing the properties of the field to be modeled. Specifically, we apply the NLFFF extrapolations not only to a very nearly force-free lower boundary (equivalent to a chromospheric vector field), but also to a lower boundary in which a vertical force acts on the field (thus emulating a photospheric vector field subject to buoyancy forces). The latter case is formally inconsistent, because it asks the NLFFF methods to seek a force-free solution based on a forced boundary. That, however, is the case in which solar physicists find themselves when using photospheric observations, such as those from the newly commissioned Hinode/SOT or from the upcoming SDO/HMI instruments. Not surprisingly, we find that this inconsistency leads to poor model field solutions, but we demonstrate that there are means to preprocess the photospheric field so that the quality of the NLFFF solutions improves considerably, at least above the chromospheric layers where the field becomes force free. As in Paper I, we find that it is important to include information on the field outside the modeled volume proper; here we represent that far field by the equivalent magnitude of the field on the top boundary and on the four side boundaries, subject to the approximation that the distant field is potential. Both of these issues need substantial further analysis before one can assess whether NLFFF modeling can yield realistic coronal fields for solar observables, but we defer that to later studies while here concentrating on what can be learned under ideal, laboratory-like conditions.

Among the other advances relative to Paper I, we note that several of the codes have in the meantime improved their performance, now enabling calculations within $320 \times 320 \times 258$ pixel volumes (improving upon the $64^{3}$-pixel domains used in Paper I). Moreover, we include an assessment of the field's topology (specifically, the field-line connectivity) in our 


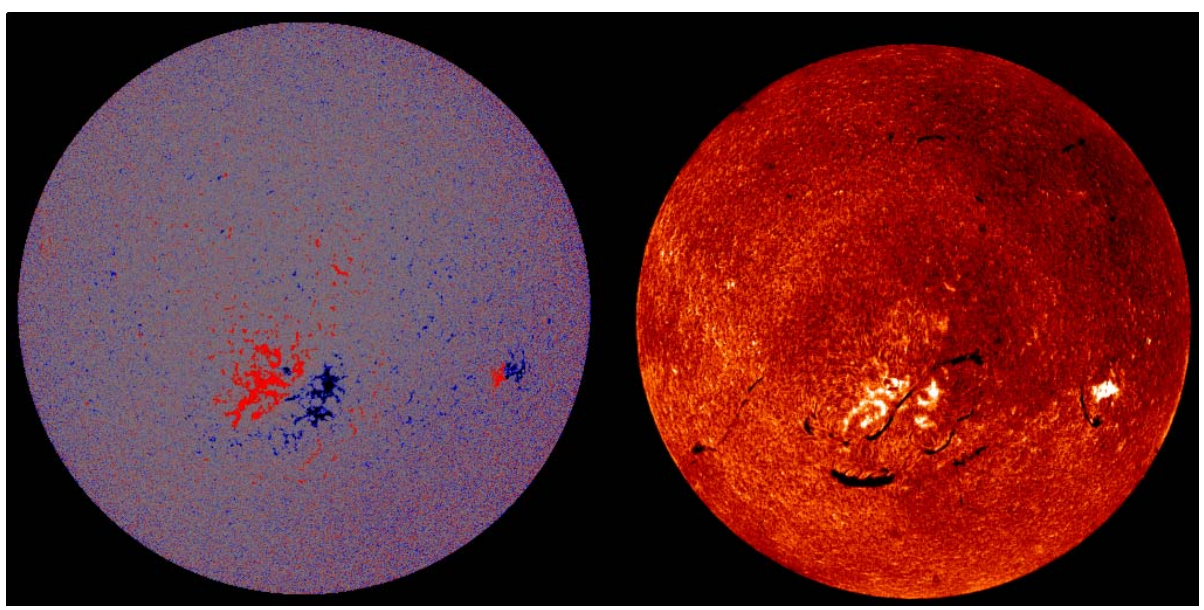

Figure 1 The MDI magnetogram from 10 October 2005 (left) shows a large, reasonably isolated active region (NOAA AR 10814) in the southern hemisphere. The corresponding H $\alpha$ image from Big Bear Solar Observatory (right) shows a filament along the polarity inversion line.

discussion of the success of the various methods. And finally, we also compare the estimates for free energy obtained with NLFFF modeling to that obtained by the magnetic virial theorem which is based on an integral over the boundary of the model volume.

\section{The van Ballegooijen Reference Model}

\subsection{Model Data}

The model data used for the tests described here were constructed by Aad van Ballegooijen. He computed a potential field in spherical coordinates using the full-disk SOHO/MDI (Scherrer et al., 1995) line-of-sight magnetogram from 10 October 2005, in which an isolated active region (NOAA AR 10814) is evident in the southern hemisphere, as shown in the left panel of Figure 1. Based on the presence of an $\mathrm{H} \alpha$ filament following the polarity inversion line of AR 10814 (as seen in the right panel of Figure 1), he inserted an S-shaped sinistral flux bundle into the potential field, producing a weakly twisted, right helical flux rope. The procedure and the boundary conditions were described in detail by van Ballegooijen (2004) and van Ballegooijen et al. (2007), while the magnetofrictional code used to relax the field to an essentially force-free state was described by van Ballegooijen, Priest, and Mackay (2000). The magnetofrictional code enforces the following boundary conditions: fixed vector potential components $A_{x}$ and $A_{y}$ at the lower boundary, periodic field at the azimuthal boundaries with currents only normal to the latitudinal side boundaries, a tangential field at the latitudinal side boundaries, and a radial field at the top.

The flux rope thus inserted is only weakly twisted; the core of the flux rope is highly sheared with respect to the overlying arcade, but the core field is untwisted. The final model contains several interesting topological features including a coronal null and its associated separatrix surface, and the S-shaped flux bundle surrounded by a quasi-separatrix layer (QSL). 
The addition of the flux bundle creates a current system in the arcade within which the field is deflected by up to 80 degrees counterclockwise away from the potential field configuration, thus reshaping the field to almost follow the direction of the underlying polarity inversion line low in the arcade. Above the current-carrying arcade, the field is deflected only slightly from the potential configuration: over the center of the arcade, the difference between the model and potential field vectors amounts to merely $\approx 5$ degrees up to top of the volume, while the magnitude of the field is increased by approximately 15 to $20 \%$ relative to the potential field. Apparently, the curved ends of the current-carrying arcade are placed such that the net effect mimics a dipole largely aligned with the magnetic axis of the active region that lies at a considerable angle relative to the main body of the arcade and associated neutral line.

Because the NLFFF codes (described in Section 3) assume a Cartesian geometry, ${ }^{1}$ the horizontal extent of the magnetogram was scaled down by a factor of 30 such that the active region spanned 1.83 degrees in both latitude and longitude. This mitigates curvature effects arising from the sphericity of the Sun.

The inserted flux rope is initially not in force balance with the surrounding arcade. In order to create force balance, the combined field is relaxed to a near force-free state using a magneto-frictional code that assumes spherical geometry and ideal-MHD in the corona (van Ballegooijen et al., 2007). To achieve more realistic magnetic fields between photospheric and chromospheric heights in the model, an upward force was applied to the field near the lower boundary during the relaxation process. This added force causes the photospheric fields to be more vertical than they would otherwise be, producing field lines that are nearly vertical immediately above the photosphere, and that fan out gradually with height (as opposed to "monopoles" that splay our immediately above the boundary plane). On the real Sun, such vertical forces are produced by magnetic buoyancy. Inside solar photospheric flux tubes, the gas pressure (and therefore the density) is lower than in the field-free surroundings, which creates a net upward buoyancy force that is balanced by a decrease with height of the magnetic tension along the tube. The upward force in the model mimics this effect.

The upward force is added only at one height in the "photosphere", at a level of one cell above the model base. In the scaled model, this corresponds to a height $r=r_{1}=10^{-4}$ solar radii. In the magneto-frictional relaxation process, the overall plasma velocity is given by

$$
\mathbf{v}=\left(f \nabla \times \mathbf{B}-v_{1} \hat{\mathbf{r}} \times \mathbf{B}\right) \times \mathbf{B} / B^{2},
$$

where $\mathbf{B}$ is the magnetic vector, and $\hat{\mathbf{r}}$ is the unit vector in the vertical direction. The first term is proportional to $(\nabla \times \mathbf{B}) \times \mathbf{B}$, the Lorentz force, and is called the magneto-frictional velocity ( $f$ is the coefficient of magneto-friction). This term is present at all heights other than the base $(r=0)$, and it is responsible for the field relaxation toward a force-free configuration. The second term represents the additional nonmagnetic force, and is present only at one height on the "photosphere", at a level of one gridpoint (approximately $10^{-4} R_{\odot}$ in our scaled down model) above the model base $\left(r=r_{1}\right)$. This additional force is proportional to $-(\hat{\mathbf{r}} \times \mathbf{B}) \times \mathbf{B} / B^{2}$, which is perpendicular to the local magnetic field $\mathbf{B}$. The quantity $v_{1}$ $\left(=0.117 \mathrm{~km} \mathrm{~s}^{-1}\right)$ is constant over the horizontal plane and independent of the magnetic field.

The velocity field $\mathbf{v}(\phi, \theta, r, t)$ in the model is used to evolve the magnetic field $\mathbf{B}(\phi, \theta, r, t)$ according to the ideal-MHD induction equation (except very early in the relaxation process when a small amount of diffusion is used for numerical reasons). Eventually

\footnotetext{
${ }^{1}$ Versions of these codes that can accommodate a spherical geometry are in development; see, e.g., Wiegelmann (2007).
} 

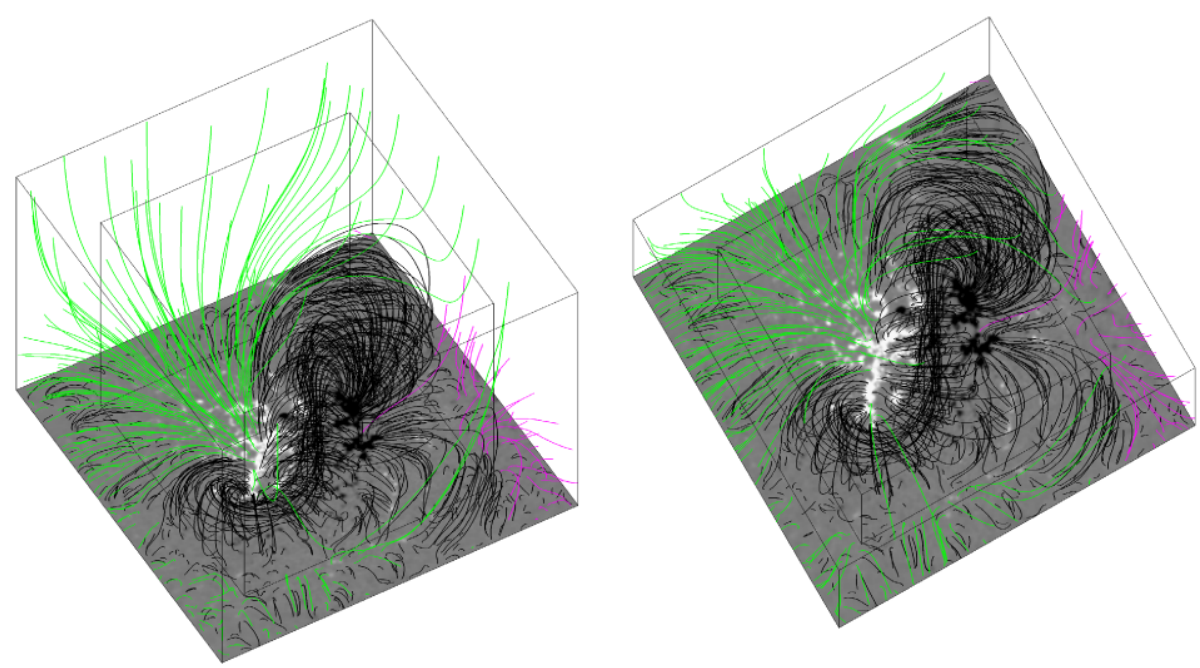

Figure 2 Representative field lines drawn through the van Ballegooijen reference model. This model was created by inserting an S-shaped flux rope into a potential-field extrapolation for the MDI magnetogram shown in Figure 1, and then using a magneto-frictional code to relax the combined field to a nearly force-free state. The cubical outline encompassing the middle of the domain demarcates the region in which the NLFFF solutions will be compared.

the field relaxes to a stationary state with $\mathbf{v} \approx 0$ everywhere. In this state, the perpendicular component of $\nabla \times \mathbf{B}$ vanishes everywhere except at $r=r_{1}$. According to the above equation, at $r=r_{1}$ we have

$$
(\nabla \times \mathbf{B})_{\perp}=\left(v_{1} / f\right) \hat{\mathbf{r}} \times \mathbf{B},
$$

so there is an additional horizontal current in regions where the magnetic field $\mathbf{B}$ has a horizontal component. This additional current produces a kink in the field lines, and forces the field lines to be more vertical in the region $r<r_{1}$.

After the relaxation process, the resulting magnetic field was interpolated onto a Cartesian grid, and a volume centered on the active region spanning 320 pixels in each horizontal direction and 258 pixels in the vertical direction was extracted. The grid spacing is $10^{-4}$ solar radii in all three directions, with the origin centered on the active region at the surface. The $x$ - and $y$-axes are defined to be parallel to the longitudinal and latitudinal directions, and the $z$-axis extends radially upward and is normal to the surface of the Sun at the origin. In what follows, we denote the $i$ th pixel in height as $z_{i}$, such that the model base is located at a height $z_{0}$, the first grid level above the base is $z_{1}$, etc. This extracted volume, through which illustrative field lines are drawn in Figure 2, is used as the reference model for the NLFFF extrapolations we describe in Section 6.

Two different heights in the Cartesian model were used as boundary conditions for the NLFFF algorithms. For the "chromospheric" case, the boundary data used as input to the NLFFF modeling codes was located at a height of $z=z_{2}$ in the reference model, where the field is largely force-free. The boundary data used in the forced "photospheric" case was taken from $z=z_{0}$, i.e., the base of the reference model, just below the height where the force was applied during the relaxation process. By using both force-free and forced test cases, we will determine to what extent the forces in the "photospheric" data set interfere with the extrapolation of the flux rope. 


\subsection{Forces and Energy in the Reference Model}

The van Ballegooijen reference model is not strictly force-free, even above the layer where the upward force is added. The residual Lorentz forces in the model result from two separate sources: (1) The original spherical geometry model is not exactly force-free because the axial flux of the flux rope is a bit too large to be securely held down by the overlying arcade. The flux rope is slowly rising, aided by numerical diffusion in the thin current layer that separates the flux rope from its surroundings. At heights above the photospheric layer, the forces computed internally by the magneto-frictional code are in the range $1-7 \%$ of the magnetic pressure gradient forces. (2) Additional forces are introduced during the calculation of the electric current density $\mathbf{J}$ from the interpolated $\mathbf{B}$ using centered differences, as would be required with real solar observations. These $\mathbf{J}$ vectors differ from the ones used originally in the magneto-frictional code, so that $\mathbf{J}$ and $\mathbf{B}$ are no longer exactly aligned. This is the main contribution to the residual force in and near the flux rope where the magnetic field varies strongly with position. For comparison, the imposed buoyancy force at layer $r_{1}$ is of order $80 \%$ of the magnetic pressure gradient force (see Figure 3, top).

The magnitudes of the forces in the model are consistent with what is known of forces on the Sun, making the model a realistic, solar-like test case for the extrapolation codes. As described above, the reference model is forced at $r=r_{1}$ to produce "flux tubes" that fan out gradually with height. Although the details of the Lorentz force on the Sun are not well known, we can compare the forces in the model to observations of the net force made by Metcalf et al. (1995). The net Lorentz force in the volume above the lower photospheric boundary can be computed from the vector field on the lower boundary as (Low, 1984)

$$
\begin{aligned}
& F_{x}=\frac{1}{4 \pi} \int_{z=z_{0}} B_{x} B_{z} \mathrm{~d} x \mathrm{~d} y, \\
& F_{y}=\frac{1}{4 \pi} \int_{z=z_{0}} B_{y} B_{z} \mathrm{~d} x \mathrm{~d} y, \\
& F_{z}=\frac{1}{8 \pi} \int_{z=z_{0}}\left(B_{z}^{2}-B_{x}^{2}-B_{y}^{2}\right) \mathrm{d} x \mathrm{~d} y,
\end{aligned}
$$

and measured relative to the magnetic pressure force:

$$
F_{p}=\frac{1}{8 \pi} \int_{z=z_{0}}\left(B_{z}^{2}+B_{x}^{2}+B_{y}^{2}\right) \mathrm{d} x \mathrm{~d} y .
$$

The net forces in the reference model are $\left(F_{x} / F_{p}, F_{y} / F_{p}, F_{z} / F_{p}\right)=(0.0,0.0,0.4)$ at the photosphere, which compares favorably to the values observed on the Sun by Metcalf et al. (1995): $(0.2,0.2,0.4)$. Hence, these integral measures of the forces in the model are consistent with what is known of real photospheric forces on the Sun.

The net force above the lower boundary can be computed routinely from solar observations using Equations (3) and (4). However, for the reference model, we can go one step further and compute the Lorentz force point-by-point $\left(\equiv f_{x}, f_{y}, f_{z}\right)$, since the full vector fields, $\mathbf{B}$ and $\mathbf{J}$, are available. Figure 3 (top) shows the average, unsigned total Lorentz force, divided by the average magnetic pressure gradient force, acting at each height in the model, and demonstrates that residual forces of at least $5 \%$ are present up to height $z=z_{30}$ in the model as a result of inserting the flux rope. The large force at the base of the model is the force that is imposed to produce flux tubes that fan out gradually with height (see Section 2.1). Both the forces at the photospheric boundary and the residual forces will be 
Figure 3 Top: Horizontal averages of the total point-by-point Lorentz force of the van Ballegooijen reference model, $\frac{1}{N} \sum\left(f_{x}^{2}+f_{y}^{2}+f_{z}^{2}\right)^{1 / 2}$, for the part of the volume containing the inserted flux rope (up to layer 30), including a 10-layer zone above that. The Lorentz force is plotted relative to the horizontally averaged magnetic pressure gradient force, $\frac{1}{N} \sum \nabla B^{2} / 2$; from layer 40 upward, the value averages about 0.04 . The inset image shows the magnitude of the Lorentz force at height $z=z_{10}$ (negative image); it shows that the Lorentz forces (averaging $\approx 5 \%$ of the magnetic pressure gradient forces at that height) are strongest within the inserted flux rope. Bottom: The energy, $E_{\mathrm{ff}}$, above each height and the net force,

$\left(F_{x}^{2}+F_{y}^{2}+F_{z}^{2}\right)^{1 / 2}$, at each height level $z$ in the reference model. The energy was computed using both one-sided and six-sided virial theorem and is plotted relative to the energy from the volume integral $B^{2} / 8 \pi$ above each height level $z$. The net Lorentz force is plotted relative to the magnetic pressure force, $F_{p}$. Only the lowest 40 height levels from the reference model are plotted. The Lorentz forces affect the virial theorem calculation up to height $z=z_{30}$.
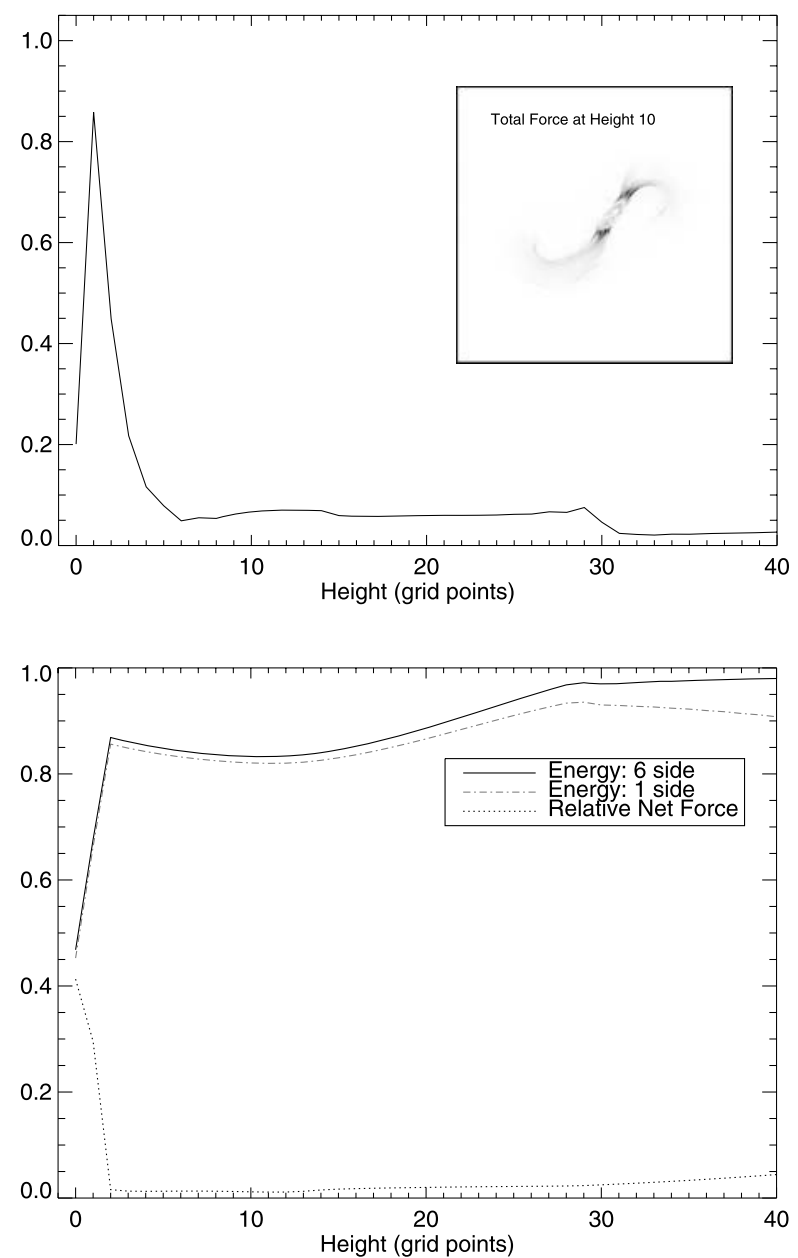

shown to have an impact on the computation of the magnetic energy from the virial theorem (discussed below) and do violate the force-free assumption made by the extrapolation algorithms, particularly in the photospheric case. Hence, this reference model presents the NLFFF algorithms with a realistic test case, and the resulting comparisons between the extrapolations and the model are indicative of how well the algorithms can be expected to perform with real solar data.

The magnetic virial theorem (e.g., Chandrasekhar and Fermi, 1953; Chandrasekhar, 1961; Molodenskii, 1969; Aly, 1984; Low, 1984; Aly, 1989; Metcalf, Leka, and Mickey, 2005) can be used to measure the energy in the magnetic field from an observation of the lower boundary of the semi-infinite volume, if the field is force-free. The virial theorem is usually written as

$$
E_{\mathrm{ff}}=\frac{1}{4 \pi} \int_{z=z \mathrm{ff}}\left(x B_{x}+y B_{y}\right) B_{z} \mathrm{~d} x \mathrm{~d} y
$$


where $B_{x}, B_{y}$, and $B_{z}$ are the horizontal and vertical components of the magnetic field, $x$ and $y$ are the horizontal coordinates on the Sun (e.g., Gary and Hagyard, 1990), and $z_{\mathrm{ff}}$ is the lower, force-free boundary.

While the virial theorem applies only for force-free fields, Equation (5) makes the additional assumption that the field falls off faster than $1 / R^{1.5}$. This constraint is obviously satisfied when the field does not connect to regions outside the observed lower boundary; however, the assumption can be relaxed if the field on the side walls of the volume of interest is known. This is helpful for the reference model which has a periodic boundary condition in one direction. The more general expression of the virial theorem (which contains Equation (5) as a special case) is

$$
E_{\mathrm{ff}}=\frac{1}{4 \pi} \int_{\delta V}(\mathbf{B} \cdot \mathbf{x})(\mathbf{B} \cdot \mathbf{n}) \mathrm{d}^{2} x-\frac{1}{8 \pi} \int_{\delta V} B^{2}(\mathbf{x} \cdot \mathbf{n}) \mathrm{d}^{2} x .
$$

Here, $\mathbf{B}$ is the vector magnetic field, $\mathbf{x}$ is the vector position, $\mathbf{n}$ is the inward normal to the surface, and the integration is done over the surface $\delta V$ bounding the volume in which the energy is contained.

Since the field on the side walls is known for the reference model, we have used both Equation (5) and Equation (6) to compute the energy in the magnetic field. The results, relative to the energy in the reference field, $\int B^{2} / 8 \pi \mathrm{d} V$, are given in Table 1 for corresponding volumes (as indicated in the table). The magnetic virial theorem, in both forms, is applied to the "photospheric" boundary, the "chromospheric" boundary and the "preprocessed" photospheric boundary (smoothed, see Sections 4 and 7.3).

The photospheric boundary is forced, violating the assumptions used to derive the virial theorem and, indeed, the computed energy is much smaller than the known energy in the model volume. The chromospheric boundary is more force-free and the virial theorem correctly recovers most of the energy in the volume, indicating that the majority of the forces lie between the photospheric and chromospheric heights in the model. The residual forces in the model above the chromosphere account for the remaining energy difference.

We also tested the virial theorem with a preprocessed photospheric boundary, using the algorithm of Wiegelmann, Inhester, and Sakurai (2006), described in Section 4. The preprocessing removes the net Lorentz forces from the boundary while maintaining the vertical field nearly unchanged. Since the new, preprocessed photospheric boundary satisfies the necessary (but not sufficient) condition that the net forces vanish, the assumptions made in the virial theorem appear to be better satisfied, resulting in a significantly better measure of the total energy in the volume above the photosphere as compared to the unpreprocessed case. It is encouraging that the preprocessing considerably improves the energy estimate from the photospheric boundary, since the forced photospheric vector field is often all that is measured, and the virial theorem is very much faster than undertaking a NLFFF extrapolation when only a measure of the energy is required.

Since the energies computed from Equations (5) and (6) are nearly identical (Table 1), we conclude that the side walls of the reference model are sufficiently far away from the central region containing most of the flux that they do not affect the virial calculations. Of course, only Equation (5) can be applied in the solar case.

Figure 3 (bottom) shows the result of applying the virial theorem sequentially at each level in the reference model. The plots show the net force and total magnetic energy above each height. The energy is plotted relative to the volume integral of $B^{2} / 8 \pi$ in the model above each height level, and the net force is plotted relative to the magnetic pressure force (Equation (4)). The virial theorem using all the walls of the model only recovers the full energy above grid point 30 , where the residual forces from the insertion of the flux rope vanish. 
Table 1 Relative energies derived from the magnetic virial theorem.

\begin{tabular}{llll}
\hline & $\begin{array}{l}\text { Chromosphere } \\
z=2: 225\end{array}$ & $\begin{array}{l}\text { Photosphere } \\
z=0: 225\end{array}$ & $\begin{array}{l}\text { Preprocessed } \\
z=0: 225\end{array}$ \\
\hline Lower boundary (Equation (5)) & 0.86 & 0.45 & 0.81 \\
All boundaries (Equation (6)) & 0.87 & 0.47 & 0.83 \\
\hline
\end{tabular}

Table 2 A summary of the algorithms used in this study, and references to their descriptions in the literature.

\begin{tabular}{lll}
\hline Author & Algorithm & Reference \\
\hline Wiegelmann & weighted optimization & Wiegelmann (2004) \\
McTiernan & optimization & Wheatland, Sturrock, and Roumeliotis (2000) \\
Valori & magneto-frictional & Valori, Kliem, and Fuhrmann (2007) \\
Wheatland & current - field iteration & Wheatland (2006) \\
\hline
\end{tabular}

Thus, the significant forces remaining in the model up to this height have an impact on the energy computed from the virial theorem. The virial energy computed from Equations (5) and (6) diverge above grid point 30 , because flux loss through the walls is more appreciable, relative to the weakening flux, higher in the model.

The net force in Figure 3 (bottom) is defined as $\sqrt{F_{x}^{2}+F_{y}^{2}+F_{z}^{2}} / F_{p}$. The relatively large net "photospheric" force at the lowest grid point is obvious, and the net force falls to near zero at the "chromospheric" grid point just above. Although the magnitude of the net force falls monotonically with height, the relative net force estimated from a surface integral over only the lower boundary slowly increases with height above the chromosphere as flux loss through the walls becomes more important. Since the net force is weak, there is no significant impact on the application of Equation (6), and the six-sided virial theorem recovers nearly the full energy in the field at all heights above $z=z_{30}$, approaching the energy to within one percent at $z \approx z_{100}$ and even closer above that.

\section{Review of the Methods}

The NLFFF extrapolation methods used to reconstruct the model flux rope are similar to those used in Paper I and are summarized in Table 2. The algorithms have undergone some modifications since Paper I, and these algorithmic differences in each author's code are described presently.

\subsection{McTiernan: Optimization}

The optimization method (Wheatland, Sturrock, and Roumeliotis, 2000) implemented by McTiernan is unchanged from Paper I; however, a different grid scheme was used to generate the results reported here. The results are obtained by interpolating the initial potential field to a grid with nonuniform $x, y$, and $z$. The grids are constant from the origin for 10 full-resolution grid points in each direction, then the grid spacing is increased by 0.02 for each point beyond that, except for the points on the outer boundary, which are chosen to match the original outer boundaries. The solution is obtained on this grid $(159 \times 159 \times 100$ 
for photosphere, and $159 \times 159 \times 99$ for chromosphere) and then interpolated back to the original $320 \times 320 \times 258$ grid. One of these calculations takes approximately 35 minutes with a $3.2 \mathrm{GHz}$ processor, and $4 \mathrm{~GB}$ of memory.

These interpolated solutions were used as a starting point for the final, full-resolution calculation in which the boundaries of the interpolated solutions were set to the boundaries of the full-resolution potential field on the outer boundaries, and the original model data on the bottom boundary, and then iterated until convergence.

\subsection{Wheatland: Current-Field Iteration}

A new implementation (Wheatland, 2006) of the current - field iteration (Grad and Rubin, 1958) has been developed, which is different from the Wheatland (2004) method used in Paper I. The new method is faster - the time taken scales as $N^{4}$, for a three-dimensional grid with $N^{3}$ points, whereas for the older method the scaling was $N^{6}$. The new method performs the field-updating part of current - field iteration via a three-dimensional Fast Fourier Transform solution of Ampere's law for a current density field constructed to implicitly satisfy the required boundary conditions on the field. The current density field is defined in both $z \geq 0$ and $z<0$. In $z \geq 0$ it is given (at iteration $k$ ) by $\alpha^{k} \mathbf{B}^{k} / \mu_{0}$, where $\mathbf{B}^{k}$ and $\alpha^{k}$ are the field and current distribution obtained at the previous iteration. In $z<0$, the current is closed via paths which mirror those in $z>0$ (see e.g., Sakurai, 1981). This procedure accounts for the speed of the method.

The current-updating step of current-field iteration is achieved via field line tracing. For each point on the computational grid, the field line threading the point is traced in both directions. If the field line leaves the side or top of the box, $\alpha$ is assigned to be zero at the point. If the field line connects to $z=0$ at both ends, $\alpha$ is assigned at the point based on bilinear interpolation of neighboring values at the positive polarity end of the field line. The code implementing the method is written in Fortran 90 and is parallelized using OpenMP for shared memory multiprocessors. Full details of the method, including applications to test cases, were given by Wheatland (2006).

For the chromospheric and photospheric data obtained from the van Ballegooijen model, the method was applied as follows. Values of $\alpha=\mu_{0} J_{z} / B_{z}$ were determined for points with $\left|B_{z}\right|>10^{-3} \times \max \left(B_{z}\right)$. Calculations were performed on the full $320 \times 320 \times 258$ grid, with the vertical grid spacing matching the horizontal spacing. Results are presented here for the field after 10 iterations, with the boundary values of $\alpha$ held fixed at the van Ballegooijenderived values at each iteration. The calculations took $\approx 12$ hours on a dual processor, dual core PC. In each case it was found that the results are not fixed points of the iteration - the field was still changing after 10 iterations. This is likely due to the boundary conditions being inconsistent with the force-free model (see Section 2.2). Minimum current-weighted angles between $\mathbf{J}$ and $\mathbf{B}$ (see Section 5) were found after about seven iterations, and thereafter the angle slowly increased.

\subsection{Wiegelmann: Weighted Optimization}

The details of the Wiegelmann method were described by Wiegelmann (2004) and summarized in our first NLFFF paper. The basic code has not been changed; however, a new multigrid (i.e., grid-refining) extension of the code has been implemented.

The method does not incorporate different grids simultaneously, but computes the solution serially on a number of different horizontal grid scales, $80 \times 80 \times 63,160 \times 160 \times$ $128,320 \times 320 \times 256$ pixels, with the results of the coarser resolution used to initialize the 
next, finer resolution. The solution from the coarser grids are interpolated onto finer grids as the initial state for the magnetic field in the computational domain of the next larger box. This gives a better starting equilibrium in the full resolution box, an improvement on an initial potential field. In practice, the multigrid version of the code is found to both converge to a lower $L$ value (see Paper I) and run faster. The code implementing the method is written in $\mathrm{C}$, and is parallelized using OpenMP for shared-memory architectures. The chromospheric solution took $2 \mathrm{~h} 15 \mathrm{~m}$ in, but the photospheric solutions took only $35 \mathrm{~m}$, not including any preprocessing. All calculations were performed on one 4-CPU node of a 2.66 GHz SGI Altix XE with 4 GB of RAM.

\subsection{Valori: Magneto-Frictional Relaxation}

The basic idea of the magneto-frictional method (see Yang, Sturrock, and Antiochos, 1986) is to include in the MHD momentum balance equation a fictitious friction that drives the system toward an equilibrium solution, under prescribed boundary conditions. As an initial condition, a potential field is used where the field at the photospheric level is substituted with the vector magnetogram. The reduced momentum equation is then integrated jointly with the ideal MHD induction equation in the interior of the box in order to relax the initial field to a force-free equilibrium as consistent as possible with the magnetogram.

The implementation of the magneto-frictional method was described in Paper I and in more detail by Valori, Kliem, and Fuhrmann (2007). The main improvement introduced in the code with respect to those papers was to change the spatial discretization from a second to a fourth order, central difference scheme, which yielded a significantly better reconstruction of the analytical test fields of Paper I. The scaling of the present implementation with the number of grid points $N$ is slightly below $N^{5}$. Large improvements in the execution time can still be obtained by using the time discretization described by Chodura and Schlüter (1981). However, such modifications are not yet included, and the $320 \times 320 \times 258$ grid points required in the test field presented here is already at the limit of the current implementation.

In order to partially correct for this limitation, two strategies were adopted. First, a strongly stretched grid in the $z$ direction was employed in order to reduce the number of grid points needed overall. The code uses a nonuniform grid in the vertical direction above grid point $61\left(z>z_{61}\right)$, so that the final volume has $320 \times 320 \times 107$ grid points. The horizontal grid is uniform. Above $z=z_{61}$, the spacing is stretched exponentially, up to a factor of 12. The extrapolation was performed on the stretched grid and then the result was interpolated on the required uniform grid.

Second, a multigrid strategy analogous to the one described in Section 3.3 is employed. In our case, the main advantage of the multigrid strategy was to shorten the overall extrapolation time. At the side and top boundaries, the tangential components of the magnetic field are extrapolated onto the boundary grid points, while the normal component is fixed at each iteration by requiring that the field remains divergence free.

The use of a heavily stretched grid and the interpolation into a uniform grid of the extrapolated field obviously lowers the accuracy of the solution and of the metrics computed below. On the other hand, they kept the execution time within acceptable limits. The code, which required $2.5 \mathrm{~GB}$ of memory, run on a $350 \mathrm{MHz}$, four-processor machine, and the execution times were 21 hours for both the chromospheric and the photospheric cases.

\section{Preprocessing}

Measurements of the photospheric magnetic vector field contain inconsistencies and noise. In particular, the magnetic field in the photosphere is not necessarily force-free and, hence, 
not consistent with the assumption of a force-free field. Wiegelmann, Inhester, and Sakurai (2006) developed a preprocessing procedure to drive the observed non-force-free data towards suitable boundary conditions for force-free extrapolation codes. We have applied the preprocessing to the photospheric layer of the van Ballegooijen reference model to test whether the preprocessed lower boundary gives a better extrapolation than the forced boundary.

To do so, we minimize a 2D functional, similar to that from Wiegelmann, Inhester, and Sakurai (2006):

$$
L=\mu_{1} L_{1}+\mu_{2} L_{2}+\mu_{3 x} L_{3 x}+\mu_{3 y} L_{3 y}+\mu_{3 z} L_{3 z}+\mu_{4} L_{4},
$$

where

$$
\begin{aligned}
L_{1}= & {\left[\left(\sum_{p} B_{x} B_{z}\right)^{2}+\left(\sum_{p} B_{y} B_{z}\right)^{2}+\left(\sum_{p} B_{z}^{2}-B_{x}^{2}-B_{y}^{2}\right)^{2}\right], } \\
L_{2}= & {\left[\left(\sum_{p} x\left(B_{z}^{2}-B_{x}^{2}-B_{y}^{2}\right)\right)^{2}+\left(\sum_{p} y\left(B_{z}^{2}-B_{x}^{2}-B_{y}^{2}\right)\right)^{2}\right.} \\
& \left.+\left(\sum_{p} y B_{x} B_{z}-x B_{y} B_{z}\right)^{2}\right], \\
L_{3 x}= & \sum_{p}\left(B_{x}-B_{x \mathrm{obs}}\right)^{2}, \\
L_{3 y}= & \sum_{p}\left(B_{y}-B_{y \mathrm{obs}}\right)^{2}, \\
L_{3 z}= & \sum_{p}\left(B_{z}-B_{z \mathrm{obs}}\right)^{2}, \\
L_{4}= & {\left[\sum_{p}\left(\Delta B_{x}\right)^{2}+\left(\Delta B_{y}\right)^{2}+\left(\Delta B_{z}\right)^{2}\right] . }
\end{aligned}
$$

The surface integrals are here replaced by a summation $\left(\sum_{p}\right)$ over all grid nodes $p$ of the bottom surface grid and the differentiation in the smoothing term is achieved by the usual five-point stencil for the 2D Laplace operator, designated by $\Delta$. Each constraint $L_{n}$ is weighted by a factor $\mu_{n}$. The first term $(n=1)$ corresponds to the force-balance condition (Equation (3)), the next $(n=2)$ to the torque-free condition. The following terms $(n=3[x, y, z])$ ensure that the optimized boundary condition agrees with the measured photospheric data, and the last term $(n=4)$ controls the smoothing.

The aim of the preprocessing procedure is to minimize $L$ so that all terms $L_{n}$ are made small simultaneously. A strategy on how to find the optimal parameters $\mu_{n}$ was described by Wiegelmann, Inhester, and Sakurai (2006). As a result of the preprocessing, we obtain a data set which has no net force or torque and, as a practical matter, the performance of the extrapolations is improved.

It is advantageous to hold the vertical field nearly fixed so that the potential field associated with the boundary is not modified, and we set $\mu_{3 z}$ to a large value and $\mu_{4}$ to a small but finite value. If the vertical field is allowed to vary, then there is flux loss and the energy in the extrapolated field may be reduced. With $B_{z}$ nearly fixed, the net Lorentz force and torque 
$\left(L_{1}\right.$ and $L_{2}$ ) are driven to zero by adjusting primarily the horizontal field. In Section 7.3 we show that some smoothing is advantageous, particularly for the NLFFF codes which rely on numerical differentiation, i.e., it is better not to set $\mu_{4}$ to zero.

\section{Figures of Merit}

To quantify how well each algorithm performed, we use four of the metrics from Paper I: the vector correlation, Cauchy-Schwartz, normalized vector error and mean vector error metrics, and supplement these with a current-weighted sine metric, a field-line divergence metric (Barnes, Leka, and Wheatland, 2006), and a free energy metric. The domain of comparison is a $224^{3}$-pixel volume centered on the active region, extending upward from height level $z=z_{2}$. As discussed in Paper I, the side and top boundary conditions affect the resulting solutions, and so we choose our domain of comparison to be a subvolume of the full computation domain.

For input field $\mathbf{B}$ (the van Ballegooijen reference model) and extrapolated field $\mathbf{b}$, the four metrics from Paper I are: the vector correlation metric

$$
C_{\mathrm{vec}} \equiv \frac{\sum_{i} \mathbf{B}_{i} \cdot \mathbf{b}_{i}}{\left(\sum_{i}\left|\mathbf{B}_{i}\right|^{2} \sum_{i}\left|\mathbf{b}_{i}\right|^{2}\right)^{1 / 2}},
$$

the Cauchy-Schwartz metric

$$
C_{\mathrm{CS}}=\frac{1}{M} \sum_{i} \frac{\mathbf{B}_{i} \cdot \mathbf{b}_{i}}{\left|\mathbf{B}_{i}\right|\left|\mathbf{b}_{i}\right|} \equiv \frac{1}{M} \sum_{i} \cos \theta_{i},
$$

the normalized vector error metric

$$
E_{\mathrm{n}}=\frac{\sum_{i}\left|\mathbf{b}_{i}-\mathbf{B}_{i}\right|}{\sum_{i}\left|\mathbf{B}_{i}\right|},
$$

and the mean vector error metric

$$
E_{\mathrm{m}}=\frac{1}{M} \sum_{i} \frac{\left|\mathbf{b}_{i}-\mathbf{B}_{\mathbf{i}}\right|}{\left|\mathbf{B}_{\mathbf{i}}\right|} .
$$

Here, the summations are over all points $i$ in the domain of comparison, $M$ is the total number of field vectors in the sum, and $\theta_{i}$ is the angle between the model and extrapolated fields at point $i$. A detailed description of how these metrics behave can be found in Paper I. We note here that $E_{\mathrm{n}}$ and $E_{\mathrm{m}}$ are sensitive to the difference in vector norm as well as angle between the two vector fields being compared, whereas $C_{\mathrm{vec}}$ and $C_{\mathrm{CS}}$ are relatively more sensitive to angle differences; hence a good metric for the former two is associated with a good metric for the latter two, but the reverse is not necessarily true.

The current-weighted sine metric is given by

$$
\mathrm{CW} \sin =\frac{\sum_{i}\left|\mathbf{J}_{i}\right| \sigma_{i}}{\sum_{i}\left|\mathbf{J}_{i}\right|}
$$

with

$$
\sigma_{i}=\frac{\left|\mathbf{J}_{i} \times \mathbf{B}_{i}\right|}{\left|\mathbf{J}_{i}\right|\left|\mathbf{B}_{i}\right|}=\left|\sin \theta_{i}\right|,
$$


where $\mathbf{J}$ is the vector current density. This metric gives the current-weighted sine between the current and the magnetic field and hence is a measure of how force-free the field is over the volume. A force-free field will have $\mathrm{CW} \sin \equiv 0$ and a non-force-free field will have $0<\mathrm{CW} \sin \leq 1$, with a smaller value indicating a more force-free field.

The field line divergence (FLD) metric (Barnes, Leka, and Wheatland, 2006) measures how well the connectivity of the field was recovered. For both the model field and the extrapolated field, field lines are initiated at each grid point on the lower boundary and traced until intersecting another boundary. If the model and extrapolated field lines end on the same face of the computational domain, a score is assigned to the point on the boundary, given by the distance between the endpoints divided by the length of the (model) field line. A single score is given to the extrapolation by determining the fraction of the area on the boundary for which the field line divergence is large ( $>10 \%$ for the results presented here). This is a slightly different score from that used by Barnes, Leka, and Wheatland (2006). Alternatively, a single score can be assigned based on the fraction of the flux for which the field line divergence is large. One advantage to this metric is that it can indicate spatially where the extrapolation has failed to recover the model field, rather than simply giving a scalar value to indicate the overall success or failure. In particular, it can indicate whether the extrapolated field successfully recovered the interesting topological features in the reference model used here, such as the coronal null and its associated separatrix surface, and/or the S-shaped flux bundle surrounded by a QSL.

The free energy metric, $E / E_{\text {pot }}$, measures the ratio of the energy in the extrapolation to the potential energy using the same boundaries. For the reference model, the potential field used was the same potential field used to start the NLFFF extrapolations (see Section 6). Since one important use of the extrapolated fields is to quantify the total and the free magnetic energy available to power solar activity, this metric is useful in determining how robust such measurements are, though the energy above the potential field is only an upper limit to the energy available to power solar activity. This metric was calculated by doing a volume integral of $B^{2} / 8 \pi$ over the domain of comparison.

\section{Coronal Models}

Each of the algorithms tested uses as its lower boundary the vector field from one $z$-level of the van Ballegooijen reference model, either "chromospheric" at $z=z_{2}$ (more force-free), "photospheric" at $z=z_{0}$ (forced), or "preprocessed" at $z=z_{0}$ (the photospheric boundary preprocessed to remove the net forces). Additionally, all algorithms used the same corresponding initial potential field for each calculation, to enable direct comparisons of the resulting free energies. The algorithms which make use of the side walls as boundary conditions used the supplied potential field at the side and top boundaries.

The potential field in the volume was computed using the normal component of the model field on all six faces of the volume. In order to compute that field, we subtract - and later add again - a uniform vector field, whose three components are given by the net flux through each pair of opposite walls, so that the residual field is flux-balanced on each pair of walls. This allows us to compute the contribution to the potential field from each pair of parallel faces using a scalar potential given by

$$
\begin{aligned}
\Phi^{l}(\mathbf{x})= & \frac{1}{\pi} \sum_{m=0}^{N_{j}-1} \sum_{n=0}^{N_{k}-1} k_{m n}^{-1} \cos \left(\frac{m \pi x^{j}}{L_{j}}\right) \cos \left(\frac{n \pi x^{k}}{L_{k}}\right) \\
& \times\left\{A_{m n}^{l+} \cosh \left(\pi k_{m n} x^{l}\right)+A_{m n}^{l-} \cosh \left[\pi k_{m n}\left(x^{l}-L_{l}\right)\right]\right\},
\end{aligned}
$$


where $j, k, l$ are a permutation of $x, y, z$,

$$
\begin{gathered}
A_{m n}^{l \pm}=\frac{\mp\left(2-\delta_{m 0}\right)\left(2-\delta_{n 0}\right)}{L_{j} L_{k} \sinh \left(\pi k_{m n} L_{l}\right)} \int_{0}^{L_{j}} d x^{j} \int_{0}^{L_{k}} d x^{k} B^{l \pm} \cos \left(\frac{m \pi x^{j}}{L_{j}}\right) \cos \left(\frac{n \pi x^{k}}{L_{k}}\right), \\
k_{m n}=\sqrt{\frac{m^{2}}{L_{j}^{2}}+\frac{n^{2}}{L_{k}^{2}}}
\end{gathered}
$$

and $B^{l \pm}$ is the normal component on the boundary at $x^{l}=0, L_{l}$. To produce the overall potential field, the summed contributions from the faces are added to the uniform field component that was subtracted earlier.

Clearly the potential field described above is not available for solar observations where only the lower boundary is measured. However, it is important for the purpose of comparison of the various NLFFF algorithms that they all start from the same potential field and that the potential field be as consistent as possible with the underlying model. This eliminates a source of confusion and variance when comparing the results of the algorithms.

The preprocessing algorithm (Section 4) was applied to the photospheric boundary to eliminate the net force (Equation (3)) and torque in the corona by modifying primarily the horizontal field. The average change in the magnetic field over the field-of-view was $3.4 \mathrm{G}$ for both $B_{x}$ and $B_{y}$ (compared to the maximum field of $230 \mathrm{G}$ ) in order to drive the force and torque integrals to zero. Since smoothing in the preprocessing does change the normal component of the field somewhat, the initial potential field was recomputed for the preprocessed photospheric field.

Each of the algorithms used the full lower boundary for extrapolation. However, as mentioned in Section 5, the metrics were applied to a much smaller subvolume so that the edges of the extrapolations were far from the subvolume used in the metric calculations. This minimizes any edge effects from the various ways the boundaries are treated in the codes. The $C_{\text {vec }}, C_{\mathrm{CS}}, E_{\mathrm{n}}, E_{\mathrm{m}}$, and CWsin metrics were applied to the horizontal pixels $48: 271$ and the vertical pixels $2: 225$ for both the chromospheric and the photospheric cases, where the pixels numbers refer to the reference model which has a horizontal dimension of 320 pixels and a vertical dimension of 258 pixels. The metrics can be directly compared between the photospheric and chromospheric cases, since the same volume was used for both, i.e., the lowest two pixels were not included in the metrics for the photospheric case. The FLD metric used field lines traced from each horizontal pixel in the range $48: 271$, though the field lines were allowed to pass through any location in the full volume. Also, the field lines for the FLD metric were started from height zero for the photospheric case, and height two for the chromospheric case. In addition, the metrics were applied to an even smaller subvolume which focuses more on the flux rope than the surrounding potential-like domain. In this case, the horizontal extent remained $48: 71$, but the vertical extent used $2: 61$ for both the photosphere and chromosphere.

\section{Results}

\subsection{Results Using the "Chromospheric" Boundary}

Overall, the NLFFF extrapolations did qualitatively quite well at recovering the flux rope present in the van Ballegooijen reference model when the algorithms use the less forced "chromospheric" data as the lower boundary. Figure 4 shows a sample of field lines for the 
Figure 4 Renderings of selected field lines for the chromospheric test case. The starting points for all field lines (the same in each panel) form a regular grid at the lower boundary. Field lines that leave the domain through either the sides or the top are colored green or magenta, depending on polarity; all others are black. The panels are in the same order as the entries in Table 3:

(a) reference model,

(b) Wiegelmann, (c) Wheatland,

(d) Valori, (e) McTiernan,

(f) potential model.

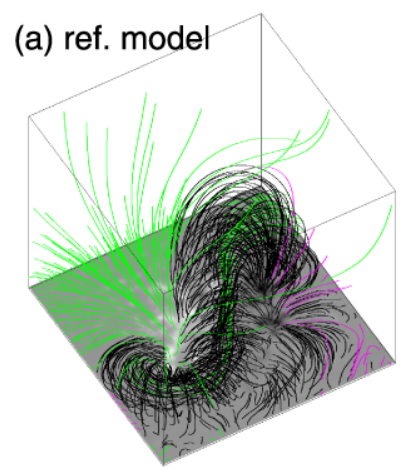

(b) Wiegelmann

(c)
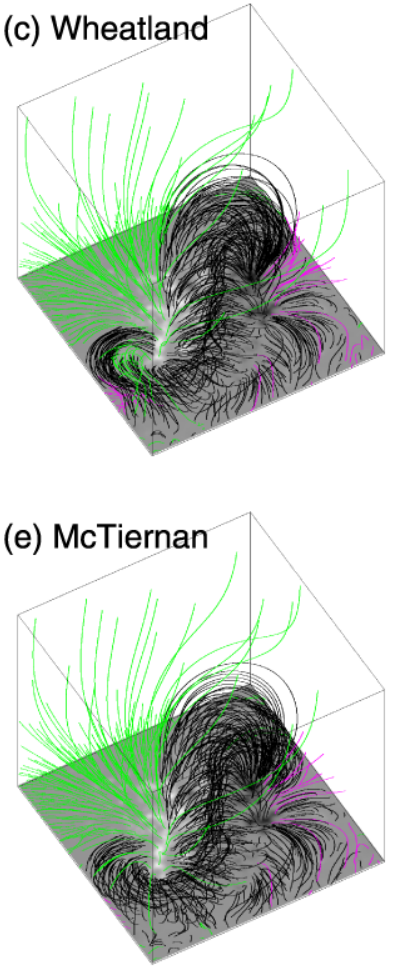

(d) Valori
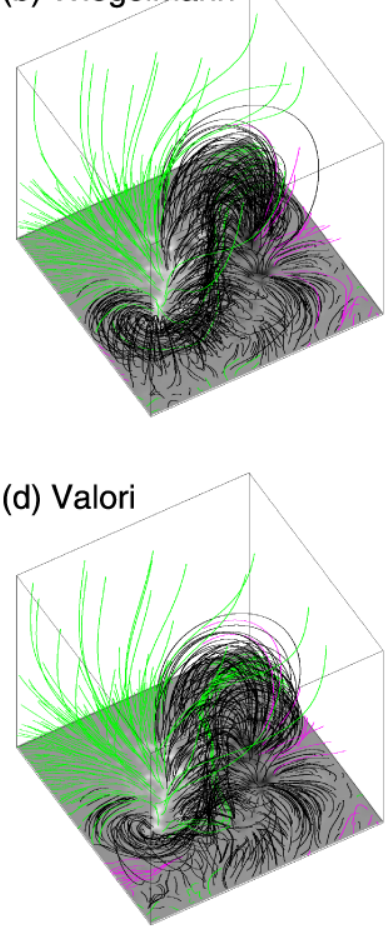

(f) potential

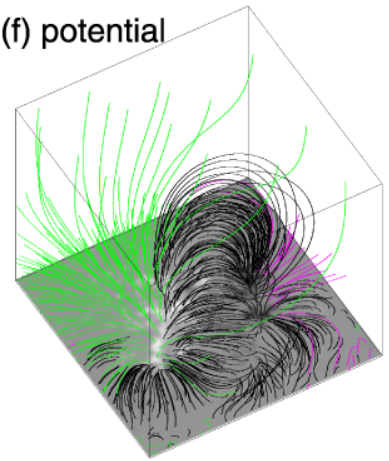

reference model, the initial potential field, and each of the extrapolations. The field lines are plotted from every fourth grid point. With the exception of the potential field, every extrapolation contains a bundle of low-lying S-shaped field lines. Hence, the algorithms were qualitatively able to recover the flux rope in the reference model, and it is quite encouraging that the algorithms are able to deal with this relatively complex test case. Overlying the S-shaped field lines in every extrapolation is an arcade of comparatively untwisted field, plus a smaller number of field lines (shown in green or red, depending on their polarity) which leave either the side or the top of the extrapolated volume. Careful inspection of the figure indicates that, although each NLFFF extrapolation produced a bundle of S-shaped field lines, the exact form of that bundle varies considerably. 
A quantitative evaluation of the methods is given in Tables 3 and 4, which list the values of the metrics, applied to two different height ranges, for each of the methods. Again, with the exception of the potential field, the extrapolations generally score quite well on most of the metrics. For example, the Cauchy-Schwartz metric in Table 3 shows that all the methods have $C_{\mathrm{CS}} \geq 0.97$. However, it should be noted that the potential field has $C_{\mathrm{CS}}=0.96$, so it is not clear that the NLFFF models are a significant improvement over the potential model based on this metric. The success of the potential model in some metrics may reflect the use of one criterion (pointwise agreement) to assess codes designed to achieve something else altogether (i.e., minimization of the Lorentz force in the volume). For the Normalized Vector Error metric, the extrapolation scores range from $0.79 \leq 1-E_{\mathrm{n}} \leq 0.89$, all of which are an improvement over the potential field's score of 0.69 . In this metric, there are clear indications that some methods perform better than others, and it is also clear that even the best method has not exactly recovered the model field.

The NLFFF codes tend to do somewhat better, in terms of the vector metrics, for the lower part of the volume than for the volume as a whole (compare the values in Table 3 for the full comparison volume with the values in Table 4 for only the lower part reaching up to twice the height of the current arcade), but the differences tend to be small: the high degree of success in reproducing the reference field apparently applies to the arcade as well as to the potential domain above it.

It is interesting to note that, although Wiegelmann and McTiernan used the same basic method for extrapolating the field, the results are significantly different, with Wiegelmann consistently scoring higher than McTiernan. Extrapolations using the Wiegelmann code, with and without the multigrid feature enabled, indicate that using the multigrid feature does affect the resulting calculation. When the multigrid feature is enabled, the solution tends to have evolved farther away from the initial potential field, and this effect might explain why the McTiernan code does not perform as well in these studies. Similarly, even though the model field was constructed using a magneto-frictional code, the extrapolation performed by Valori (also using a magneto-frictional code) did not score as highly as the extrapolation performed by Wiegelmann. Thus we conclude that the implementation of the method can be as important as the underlying approach.

Also note that, based on the current-weighted sine metric, the model field is not forcefree, i.e., CWsin is substantially different from zero. As discussed in Section 2.2, the model does contain residual forces up to height $z=z_{30}$.

The energy metric gives quite an encouraging result. All the NLFFF extrapolations perform significantly better than the potential field, and several of them have energies that are extremely close to the model field energy. This is particularly encouraging, since the energy is a physically interesting quantity. All the algorithms did at least as well as the magnetic virial theorem applied to the chromospheric boundary for which $E / E_{\text {pot }}$ is 1.15 .

However, the field line divergence metric indicates that even the best performing of the methods did not correctly reproduce all the details of the flux bundle. Figure 5 shows the location of intersections of QSLs with the chromospheric boundary, as determined using the norm of the displacement gradient tensor (Priest and Démoulin, 1995), and the FLD metric for the Wiegelmann extrapolation. For the chromospheric boundary, most of the field lines are successfully reproduced, at the $10 \%$ level, including field lines surrounding the coronal null.

For the Wiegelmann result, there are small (of order one grid point) discrepancies in the location of some QSLs, which appear as very thin bands of very large FLD, such as the one running from $(x, y) \approx(0,-75)$ to $(x, y) \approx(50,-25)$; this QSL separates field lines that connect to the strong positive polarity region from ones which connect to the second, 
Table 3 Metrics for each extrapolation code applied to the three lower boundaries $(z=2: 225)$.

\begin{tabular}{|c|c|c|c|c|c|c|c|}
\hline Model & $C_{\mathrm{vec}}$ & $C_{\mathrm{CS}}$ & $1-E_{\mathrm{n}}$ & $1-E_{\mathrm{m}}$ & CWsin & $E / E_{\text {pot }}$ & 1-FLD (area/flux) \\
\hline \multicolumn{8}{|c|}{ Chromospheric boundary (Figure 4) } \\
\hline Reference model & 1.00 & 1.00 & 1.00 & 1.00 & 0.10 & 1.34 & $1.00 / 1.00$ \\
\hline Wiegelmann & 1.00 & 0.99 & 0.89 & 0.73 & 0.11 & 1.34 & $0.85 / 0.76$ \\
\hline Wheatland & 0.95 & 0.98 & 0.79 & 0.70 & 0.15 & 1.21 & $0.51 / 0.63$ \\
\hline Valori & 0.98 & 0.98 & 0.84 & 0.71 & 0.15 & 1.25 & $0.56 / 0.58$ \\
\hline McTiernan & 0.97 & 0.97 & 0.80 & 0.69 & 0.22 & 1.15 & $0.76 / 0.70$ \\
\hline Potential solution & 0.85 & 0.96 & 0.69 & 0.67 & - & 1.00 & $0.47 / 0.57$ \\
\hline \multicolumn{8}{|c|}{ Photospheric boundary (Figure 7) } \\
\hline Reference model & 1.00 & 1.00 & 1.00 & 1.00 & 0.10 & 1.52 & $1.00 / 1.00$ \\
\hline Wiegelmann & 0.93 & 0.96 & 0.62 & 0.61 & 0.38 & 0.76 & $0.56 / 0.41$ \\
\hline Wheatland & 0.86 & 0.95 & 0.66 & 0.63 & 0.20 & 1.01 & $0.33 / 0.48$ \\
\hline Valori & 0.92 & 0.96 & 0.53 & 0.59 & 0.28 & 0.36 & $0.35 / 0.29$ \\
\hline McTiernan & 0.91 & 0.96 & 0.63 & 0.63 & 0.47 & 0.75 & $0.56 / 0.46$ \\
\hline Potential solution & 0.85 & 0.95 & 0.66 & 0.64 & - & 1.00 & $0.41 / 0.53$ \\
\hline \multicolumn{8}{|c|}{ Photospheric preprocessed boundary (Figure 10) } \\
\hline Reference model & 1.00 & 1.00 & 1.00 & 1.00 & 0.10 & 1.52 & $1.00 / 1.00$ \\
\hline \multicolumn{8}{|l|}{ Wiegelmann } \\
\hline Smoothed & 0.98 & 0.97 & 0.77 & 0.65 & 0.26 & 1.18 & $0.24 / 0.48$ \\
\hline Unsmoothed & 0.95 & 0.96 & 0.69 & 0.63 & 0.30 & 0.97 & $0.60 / 0.51$ \\
\hline \multicolumn{8}{|l|}{ Wheatland } \\
\hline Smoothed & 0.88 & 0.96 & 0.69 & 0.65 & 0.11 & 1.03 & $0.22 / 0.47$ \\
\hline Unsmoothed & 0.82 & 0.93 & 0.59 & 0.56 & 0.38 & 1.08 & $0.13 / 0.26$ \\
\hline
\end{tabular}

weaker band of positive polarity in the south-west corner of the field of view. These discrepancies represent a very small fraction of the area (and flux), and therefore do not contribute significantly to the overall score. However, there are larger patches of disagreement in the vicinity of the flux bundle. Although the existence of the flux bundle has been recovered, the exact nature of at least some of the field lines within it has not, perhaps as a result of the residual forces discussed in Section 2.2.

To understand the differences in the field line connectivity in and around the flux bundle, some illustrative field lines are shown in Figure 6 for the Wiegelmann result. Most of these field lines have been chosen to originate in the area with a large FLD, and are not therefore representative of the overall performance of the extrapolation, but do show where the extrapolation has difficulty in recovering the connectivity of the field. There are clearly several different flux domains which have not been recovered well. In the flux bundle, there are model field lines which are not in fact $\mathrm{S}$-shaped but rather intersect the chromospheric boundary close to the neutral line, and thus constitute only half an S. In some cases, the corresponding extrapolated field line dips close to the boundary, but does not intersect it, resulting in a full $S$ and a very different connectivity. There are also field lines which lie outside of the flux bundle, in which the model and extrapolated field lines slowly but steadily diverge from each other, resulting in moderate changes in the connectivity. 
Table 4 Metrics for each extrapolation code applied to the chromospheric and un-preprocessed photospheric boundaries $(z=2: 61)$.

\begin{tabular}{|c|c|c|c|c|c|}
\hline Model & $C_{\mathrm{vec}}$ & $C_{\mathrm{CS}}$ & $1-E_{\mathrm{n}}$ & $1-E_{\mathrm{m}}$ & CWsin \\
\hline \multicolumn{6}{|c|}{ Chromospheric boundary (Figure 4) } \\
\hline Reference model & 1.00 & 1.00 & 1.00 & 1.00 & 0.10 \\
\hline Wiegelmann & 1.00 & 0.99 & 0.94 & 0.89 & 0.11 \\
\hline Wheatland & 0.95 & 0.95 & 0.79 & 0.76 & 0.15 \\
\hline Valori & 0.98 & 0.96 & 0.86 & 0.81 & 0.15 \\
\hline McTiernan & 0.97 & 0.95 & 0.81 & 0.76 & 0.22 \\
\hline Potential solution & 0.85 & 0.90 & 0.66 & 0.68 & - \\
\hline \multicolumn{6}{|c|}{ Photospheric boundary (Figure 7) } \\
\hline Reference model & 1.00 & 1.00 & 1.00 & 1.00 & 0.10 \\
\hline Wiegelmann & 0.93 & 0.92 & 0.58 & 0.58 & 0.37 \\
\hline Wheatland & 0.85 & 0.90 & 0.63 & 0.65 & 0.20 \\
\hline Valori & 0.94 & 0.90 & 0.46 & 0.50 & 0.26 \\
\hline McTiernan & 0.91 & 0.91 & 0.59 & 0.60 & 0.47 \\
\hline Potential solution & 0.85 & 0.89 & 0.63 & 0.65 & - \\
\hline
\end{tabular}
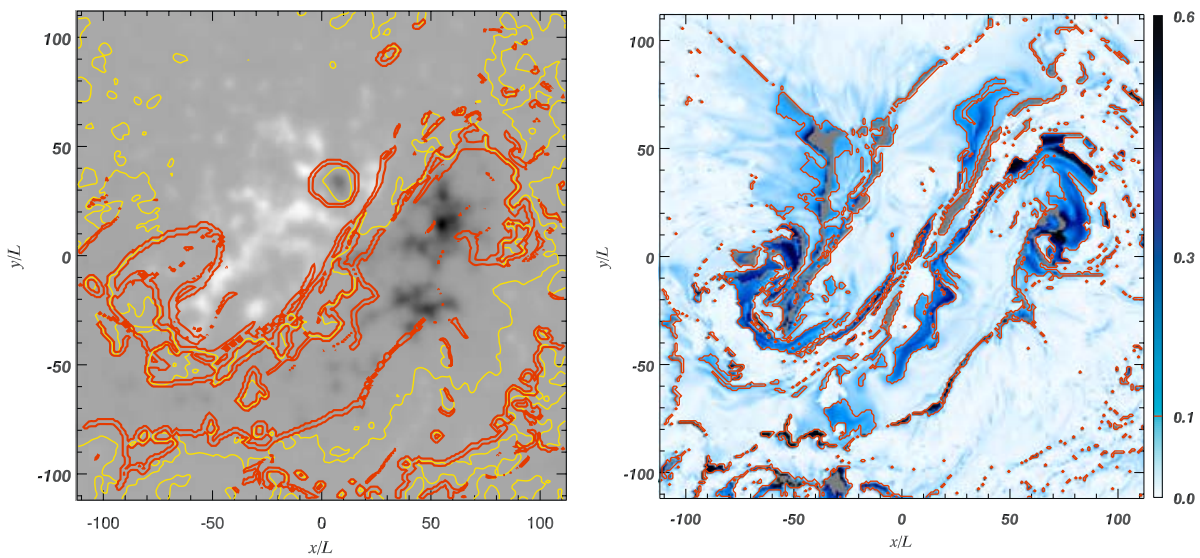

Figure 5 Left: intersection of QSLs with the chromospheric boundary (red), as determined using the norm of the displacement gradient tensor, shown superimposed on the normal field on the boundary (gray scale, with polarity inversion line in yellow). Right: the FLD metric for the Wiegelmann extrapolation. The value of the field line divergence is shown on a blue scale for field lines which end on the same wall of the computational domain, regions where field lines end on different walls are shown in gray, and a red contour highlights the $10 \%$ level. Most of the field lines, as well as the coronal null, are successfully reproduced, but there are patches of disagreement where the flux bundle intersects the boundary.

In summary, the FLD metric indicates that while the morphology of the field has been recovered, the field connectivity has not been recovered completely. This is likely to be harder, but possibly more useful, for example when determining changes in connectivity due to reconnection. 

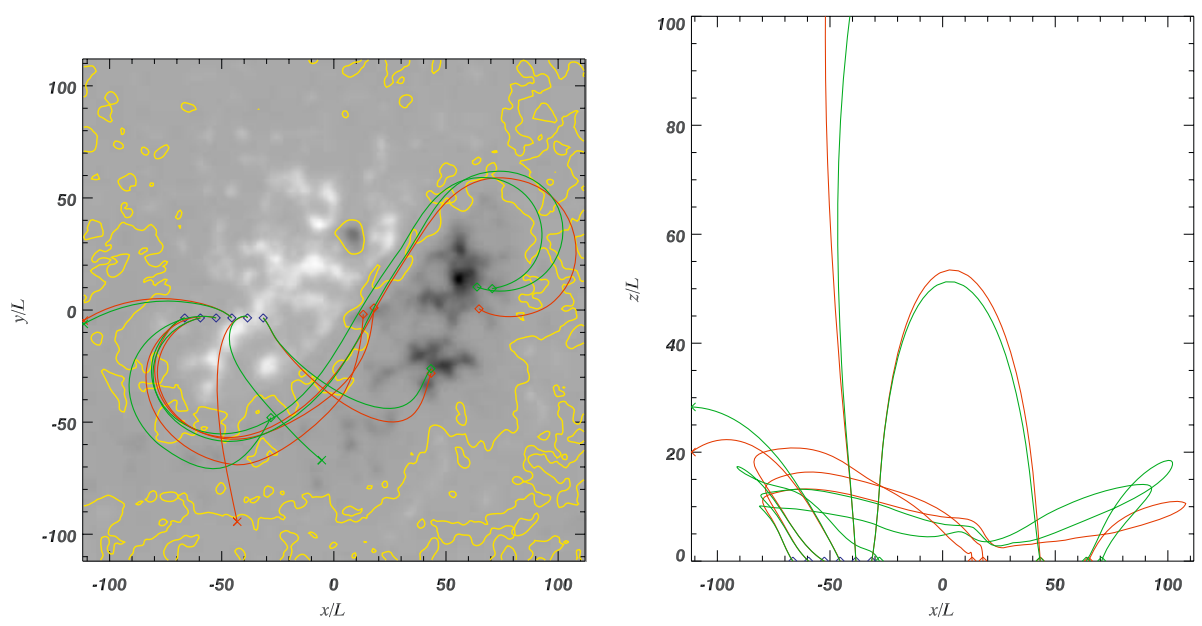

Figure 6 Sample field lines from the model field (red) and the Wiegelmann extrapolation (green) seen from above (left) and from the south (right). The vertical scale on the right panel has been expanded since most of the field lines are low-lying, which exaggerates differences in the vertical direction.

\subsection{Results Using the "Photospheric" Boundary}

Qualitatively, the algorithms did not recover the flux rope from the van Ballegooijen reference model as well when presented with the forced "photospheric" lower boundary. There is a hint of the flux rope in some of the field lines (Figure 7), but none of the algorithms were able to recover the small-scale details. Since the photospheric boundary is forced, and hence inconsistent with the assumption made by the NLFFF extrapolation codes, this is perhaps not surprising. However, since the forced photospheric field is often the only data available for extrapolations, it is important to understand quantitatively how the extrapolation algorithms perform when applied to a forced dataset.

Quantitatively, each of the algorithms scores worse on the metrics in this case than in the chromospheric case (Tables 3 and 4). For example, examining the vector correlation in Table 3, all the NLFFF extrapolations previously scored at least 0.95 , whereas now only the best performing algorithm is able to approach that level; on the Normalized Vector Error, several of the algorithms even score lower than the potential field. Indeed, there is little improvement over the potential field for most of the methods on most of the metrics. Interestingly, the distinct ways in which the codes deal with the forced lower boundary causes them to have different relative merits in different domains of the volume. For example, Valori's solution (in Figure 7d) shows a flux rope under the arcade where Wheatland's (in Figure 7c) shows no such structure at all, whereas the vector metrics computed for the full volume (in Table 3) are better for Wheatland's solution than for Valori's for five of the six metrics. Also, Valori's method scores better in the lower portion of the comparison volume (Table 4) than in the full comparison volume (Table 3), indicating that the flux rope is better recovered than the overlying potential-like domain. From the perspective of the magnetic free energy, however, none of the methods leads to an estimate that is significantly above that of a simple potential field. Note that the metrics for the photospheric case were computed within the same volume as for the chromospheric case, i.e., the two lowest levels in the photospheric extrapolations were ignored in the calculation of the metrics, with the exception of the FLD metric. 
Figure 7 As Figure 4, but for the photospheric test case.

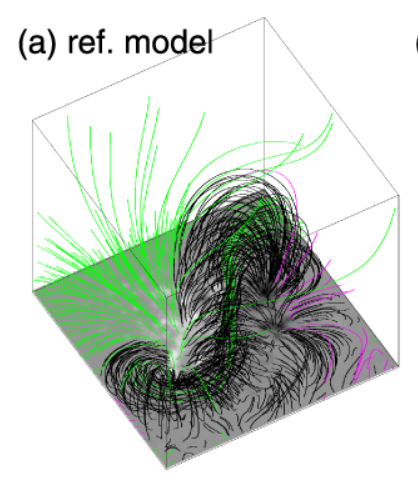

(b) Wiegelmann

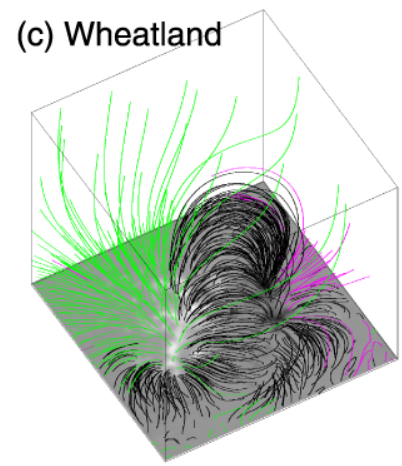

(d) Valori

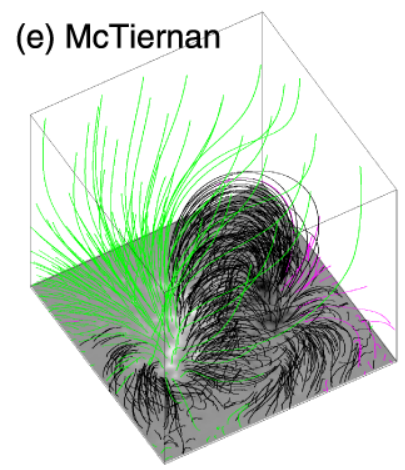

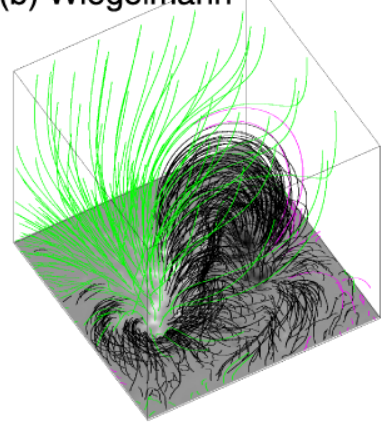

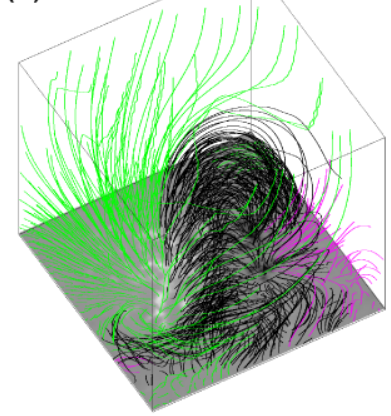

(f) potential

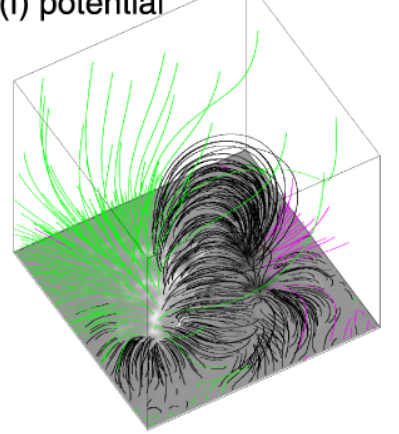

The energy metric, $E / E_{\text {pot }}$, varies widely between the algorithms, indicating that the algorithms were not able to recover the energetically significant regions in the model. In fact, some of the algorithms recover less energy than the potential field. This demonstrates that the codes do not perform well when they are based on a field with substantial, buoyancylike forces acting on the lower boundary. This is hardly surprising, since such a forced vector field is intrinsically inconsistent with the NLFFF model assumption that the field is forcefree everywhere. Most of the algorithms still do better than the virial theorem which has an $E / E_{\text {pot }}$ score of 0.60 , though we should not expect the virial theorem to provide useful results for the forced photospheric boundary. 
For the photospheric boundary data, it is the optimization and magneto-frictional methods which produce fields with energies substantially less than the potential field energy $E_{\text {pot }}$. At face value this is at odds with the minimum energy theorem (also known as Thomson's principle), which states that the potential field is the lowest energy magnetic field in a volume subject to a prescribed normal component of the field on the boundary (Sakurai, 1979; Priest, 1984). The minimum energy theorem holds for a magnetic field that satisfies $\nabla \cdot \mathbf{B}=0$ in a bounded volume. Hence, the theorem can be violated if this does not hold in all of the volume (including the boundary). If the codes do not succeed in finding the field inside the volume which is consistent with the vector magnetogram at the boundary, then the theorem may not apply.

The magneto-friction and optimization codes both try to minimize $|\mathbf{J} \times \mathbf{B}|$, and, when faced with the forced lower boundary, the codes tend have several options to achieve this: (a) align $\mathbf{J}$ and $\mathbf{B}$, (b) make $|\mathbf{B}|$ small, and/or (c) make $|\mathbf{J}|$ small. In the magneto-frictional code, the field strongly decreases above the lower boundary during the iteration. In the optimization code, the field is near potential above the lower boundary indicating the current is minimized. Both effectively introduce an unphysical boundary layer immediately above the forced lower boundary and this is the origin of $E<E_{\text {pot }}$.

The optimization and magneto-frictional methods allow departures from $\nabla \cdot \mathbf{B}=0$ in the vector fields being evolved. In fact, the starting point for the field evolution in each case is a potential field in the volume together with the photospheric data on the lower boundary. This choice introduces a nonzero $\nabla \cdot \mathbf{B}$ at the lower boundary because the vertical gradient $\partial B_{z} / \partial z$ associated with the potential field is not equal to the sum of the transverse gradients $-\partial B_{x} / \partial x-\partial B_{y} / \partial y$ associated with the photospheric boundary data (note that this implies that $\nabla \cdot \mathbf{J}$ may also not be 0 near the boundary where currents are computed from inconsistent gradients in the field). The optimization and magneto-frictional methods then each evolve the field in such a way as to attempt to reduce the value of $|\nabla \cdot \mathbf{B}|$ and thereby, indirectly, $|\nabla \cdot \mathbf{J}|$. The optimization method minimizes a volume integral involving $|\nabla \cdot \mathbf{B}|^{2}$, and the magneto-frictional method uses a form of divergence cleaning. The endpoint of the field evolution in each case should satisfy $\nabla \cdot \mathbf{B}=0$ (and $\nabla \cdot \mathbf{J}=0$ ) to a high degree of fidelity. However, if the boundary conditions are inconsistent with the force-free model, as is the case with the photospheric test case, then the endpoint of the evolution may not satisfy $\nabla \cdot \mathbf{B}=0$ or $\nabla \cdot \mathbf{J}=0$ very closely. The Wheatland current - field iteration procedure, by contrast, works with a vector potential at each iteration, so that the divergence-free condition should always be satisfied (up to truncation error).

A metric that measures the extent to which $\nabla \cdot \mathbf{B}$ is zero for each method is shown in Table 5. The metric $\left(\left\langle\left|f_{i}\right|\right\rangle\right)$ is the unsigned mean, over the comparison volume, of the quantity $f_{i}$, defined by Wheatland, Sturrock, and Roumeliotis (2000):

$$
f_{i}=\frac{(\nabla \cdot \mathbf{B})_{i}}{6|\mathbf{B}|_{i} / \Delta x},
$$

where the index $i$ varies over each element in the solution and $\Delta x$ is the grid spacing. A smaller value of $\left\langle\left|f_{i}\right|\right\rangle$ indicates that $\nabla \cdot \mathbf{B}$ is closer to zero over the volume. Inspection of $\left\langle\left|f_{i}\right|\right\rangle$ for each of the methods supports our interpretation: the metric for the photospheric data is much larger for the optimization and magneto-frictional methods when compared with the Grad-Rubin method since the Grad-Rubin method works directly with the vector potential.

The FLD metric indicates that the field connectivity is not well recovered when the NLFFF algorithms are applied to the photospheric boundary. The FLD metric for the Wiegelmann extrapolation is shown in Figure 8 as an example. Unlike the chromospheric 
Table 5 Values of the unsigned mean $\left\langle\left|f_{i}\right|\right\rangle\left(\times 10^{-4}\right)$ of the divergence metric (as defined in Equation (18)) for the field extrapolation models based on the chromospheric or photospheric lower boundaries with and without preprocessing.

\begin{tabular}{|c|c|c|c|c|c|c|}
\hline \multirow[t]{2}{*}{ Model } & \multicolumn{2}{|c|}{ Chromosphere } & \multicolumn{2}{|c|}{ Photosphere } & \multicolumn{2}{|c|}{ Preprocessed photosphere } \\
\hline & $z=2: 225$ & $2: 61$ & $2: 225$ & $2: 61$ & Smoothed & Unsmoothed \\
\hline Reference model & 1.87 & 4.04 & 1.87 & 4.04 & & \\
\hline Wiegelmann & 2.39 & 6.34 & 8.63 & 31.2 & 5.00 & 11.2 \\
\hline Wheatland & 0.20 & 0.70 & 0.92 & 2.82 & 0.18 & 2.7 \\
\hline Valori & 2.86 & 9.90 & 16.0 & 13.2 & & \\
\hline McTiernan & 2.39 & 8.75 & 9.45 & 35.7 & & \\
\hline Potential solution & 0.040 & 0.15 & 0.020 & 0.076 & & \\
\hline
\end{tabular}
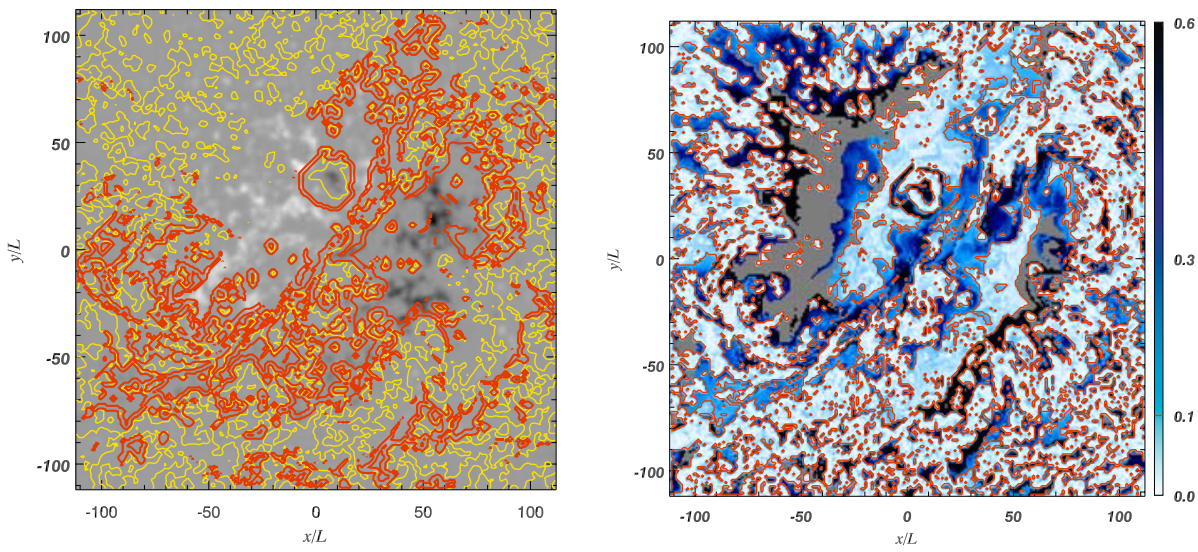

Figure 8 QSLs and the FLD metric for the Wiegelmann extrapolation from the photospheric boundary in the same format as Figure 5. The many small changes in the polarity of the field on the boundary result in a much greater number of QSLs, with a corresponding large number of different flux domains. Many of these QSLs are not well recovered by the extrapolation. More importantly, even the main features of the model field are not well reproduced. The coronal null point is present in the extrapolation, but the location of the separatrix surface is different from the model field, while the S-shaped field lines are largely absent.

case, much of the weak field area is not successfully recovered. This may not be a result of the forces on the boundary so much as it is a result of the small scale structure in the field. However, the extrapolation also does poorly in reproducing the more interesting features of the model. A coronal null point is still present, but the extrapolation did not accurately recover the location of the separatrix surface, as is evident by the ring of large FLD centered at $(x, y) \approx(10,30)$. As in the chromospheric case, the S-shaped field lines are also not well recovered; in this case, the S-shaped field lines are largely absent, in contrast to the chromospheric case, in which the field lines were qualitatively the correct shape, but did not typically intersect the boundary in the right place (compare Figure 6 with Figure 9).

It is interesting to note from the CWsin metric that each of the algorithms produced a field that is considerably less force-free than the van Ballegooijen model. Recalling that the metrics do not include the lowest two heights, it is clear that the algorithms deal with the forced lower boundary, which is inconsistent with the force-free assumption made in the 

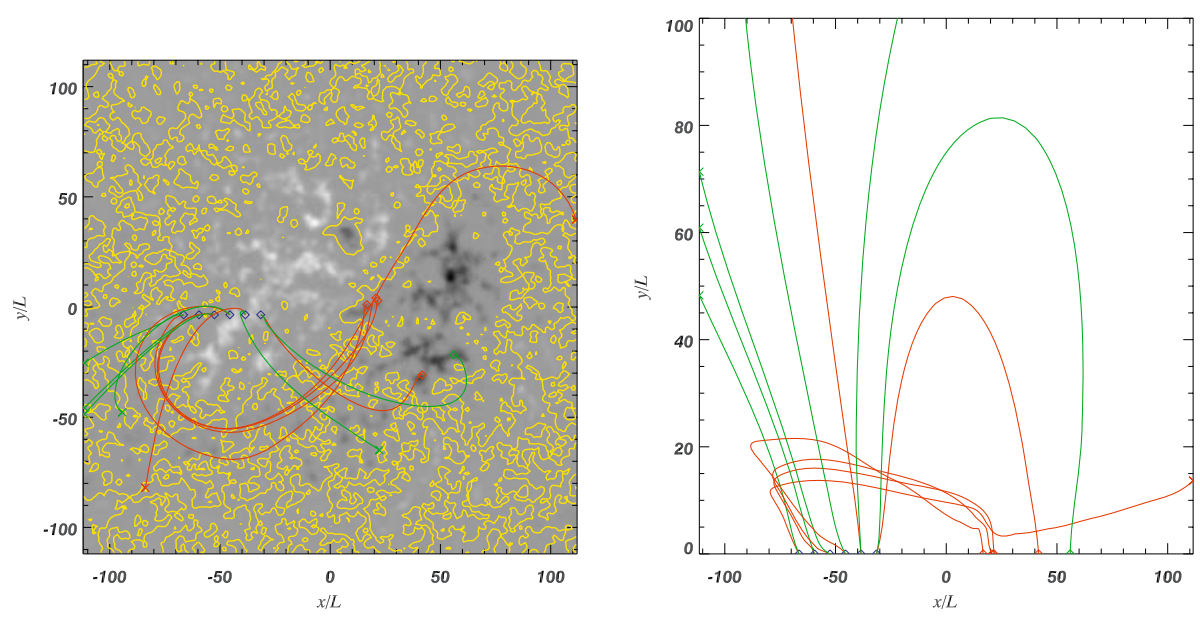

Figure 9 Sample field lines for the photospheric boundary in the same format as Figure 6.

extrapolations, by distributing the forces into the volume rather than leaving them confined to the lower layers as in the reference model.

In summary, when dealing with a forced lower boundary, one must be very cautious in applying the extrapolation algorithms as the forces may affect the NLFFF solutions appreciably. The algorithms were not able to recover the details of the flux rope when presented with the forced photospheric lower boundary.

\subsection{Results using the Preprocessed "Photospheric" Boundary}

Given the somewhat discouraging results when the NLFFF algorithms were applied to the photospheric boundary, we now consider what can be done to make better use of forced boundary data.

Although the algorithms were not able to recover the flux rope from the forced photospheric data as well as it was recovered with the chromospheric data, it is possible to remove the net photospheric forces using the preprocessing algorithm of Wiegelmann, Inhester, and Sakurai (2006). When the "photospheric" lower boundary is preprocessed, the flux rope is again recovered, at least qualitatively (Figure 10). Hence it does appear possible to recover the field morphology from a forced data set with preprocessing. Note that we have extrapolated from the preprocessed field using the Wiegelmann code and the Wheatland code, since these are very different implementations and, hence, representative of the improvements that are possible with preprocessing.

The metrics for the preprocessed boundary are uniformly improved over the unpreprocessed case, though they do not match the quality of the metrics for the chromospheric case (Table 3). So, the preprocessing does improve the extrapolations to the point that the field from the van Ballegooijen reference model is well recovered visually, but it does not fully correct for the impact of the Lorentz forces. Nevertheless, the preprocessing does allow the extrapolation algorithms to recover much of the flux rope structure and its application to photospheric data sets is thus quite encouraging.

In fact, while it is clear from Figure 10 that the flux rope can be recovered from the preprocessed boundary, it is by no means inevitable. When the Wiegelmann code without multigridding, the algorithm used in Paper I, is applied to the preprocessed data, the flux 
Figure 10 Field lines from the Wiegelmann and Wheatland solutions for the preprocessed photospheric test cases, both starting from the same potential field solutions and using preprocessed lower boundary data with (left column) and without (right column) smoothing. (a) Wiegelmann smoothed BC

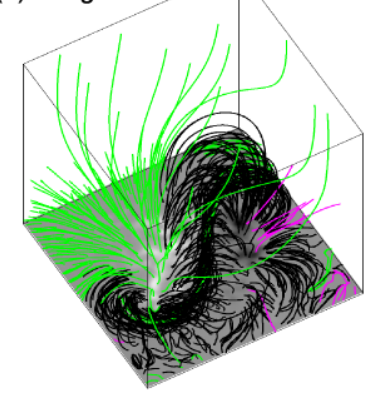

(c) Wheatland smoothed BC

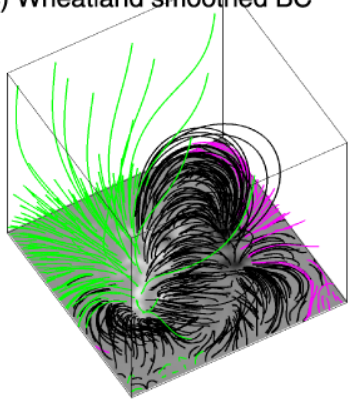

(b) Wiegelmann unsmoothed BC

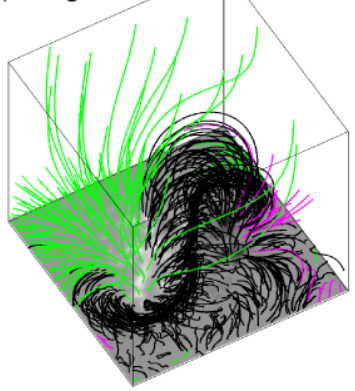

(d) Wheatland unsmoothed BC

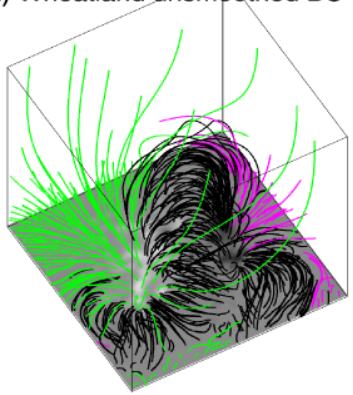

rope is not recovered and the extrapolation is very potential-like in appearance. Note that the multigridded code (Section 3.3) finds a value of $L_{w}$ (Equation (11) in Paper I) which is $6 \%$ lower than without multigridding, indicating that the solution with the flux rope is a better global minimum of the Lorentz force and field divergence than the solution without the flux rope. This sensitivity to the particulars of the algorithm implementation should serve as a caution that NLFFF modeling without coronal information for comparison, such as observations of X-ray or EUV loops, can be ambiguous.

As reported in Paper I, the NLFFF algorithms can be sensitive to the initial starting condition. We initialized the Wiegelmann and Wheatland codes from the same type of potential field initial condition that was used in the unpreprocessed cases, though the vertical field was modified somewhat by smoothing in the preprocessing so the potential field was also modified. For comparison, we also started from a potential field computed using the unpreprocessed lower boundary, but the results were not sensitive to the initial condition in this case.

The preprocessing of the non-force-free "photospheric field" aims to minimize the net forces and torques while remaining compatible with the observed line-of-sight (here normal) component of the field. As mentioned in Section 4, it is advantageous to hold the vertical field nearly fixed during the preprocessing so that the potential field associated with the boundary is not modified. To the extent that the vertical field is modified, energy can be lost in the extrapolated field in comparison to the model field. The preprocessing as proposed by Wiegelmann, Inhester, and Sakurai (2006) includes a smoothing term to dampen the highest frequency variations (including instrumental noise) in the lower-boundary vector field. This smoothing turns out to be quite important in evaluating our comparison metrics. However, since the smoothing will modify the vertical field, it must be applied carefully.

The consequences of the smoothing become clear in this thought experiment: Highfrequency noise in a vector-magnetogram (reflecting, among others, instrumental effects, 
remapping biases, or real deflections of the photospheric field under the influence of granulation and other flows) will introduce fine-scale currents of random directions in close proximity. A potential magnetic field high above a magnetogram with small errors in the line-of-sight field would not be affected by that noise as the contribution to the field by such high-order multipole sources is limited to the near field, if most of the small-scale currents close low in the volume. An ideal NLFFF field, however, will carry these electrical currents from one footpoint of the magnetic field lines to the other, thus potentially filling the entire model volume with small-scale currents of random directions.

The consequence of this is not only that maps of the electrical currents at any height will show substantial pepper-and-salt noise (as shown by the example in Figure 11 where these currents are shown for level $z_{4}$ ), but also that the NLFFF solution will have perturbations about the ideal vector field. Most field lines may still connect through the volume as they would in the noise-free case (see Figure 10). The vector perturbations do, however, cause a drop in the metrics.

This drop is seen in our test cases in Table 3, by comparing the metrics for the preprocessed photospheric field with and without smoothing. We suspect that there is an additional cause for a lowered metric in at least one of the algorithms: in Paper I, we pointed out that if an iteration was started from an initially random vector field throughout the volume, the Wiegelmann optimization code did not converge as well as in the case that the iteration was started from a potential field (Table III in Paper I). It is likely that starting from a noisy footprint vector field, the code may not converge as well as in the noise-free case, causing the metric to be lowered even more.

For the preprocessed photosphere, the virial theorem gives an $E / E_{\text {pot }}$ of 0.88 (unsmoothed) or 1.07 (smoothed), which compares favorably to the Wiegelmann extrapolation which obtains 0.97 and 1.18. Unfortunately, neither compares well to the correct value of 1.52. However, the significance of this energy deficit depends on the physical interpretation of the preprocessed field. By smoothing and removing the net Lorentz force, the preprocessed field becomes "chromospheric-like". Obviously, it is not the same as the chromospheric field in the reference model, but it is more like a chromospheric field than the forced photospheric field. In order to test this hypothesis, we compare the energy in the extrapolation to the energy computed in the reference model only for the chromosphere and above, thus mimicking the fact that the preprocessing effectively "removes" the forced layer. This is implemented by a two-pixel offset between the preprocessed extrapolation and the model to account for the height of the forced layer. The Wiegelmann solution for the smoothed, preprocessed lower boundary computed for heights $z_{0}<z<z_{223}$ recovers $96 \%$ of the energy in the reference model computed for heights $z_{2}<z<z_{225}$ with $E / E_{\text {pot }}=1.46$, a much more encouraging number than the 1.18 quoted above. Whether or not this is a general result will require further study (see Wiegelmann et al., 2008).

We do not pursue preprocessing further here, but stress that studies of optimal parameters (the $\mu$ 's in Section 4) and the physical interpretation of the preprocessed field need to be carried out. In particular, there is a trade-off between holding the vertical field fixed and smoothing, as presently implemented. Optimized preprocessing may substantially improve the ability of the NLFFF algorithms to recover the coronal field morphology, and is likely to enable a better and faster convergence, lead to less noise on maps of large electrical current systems, and improve estimates of the free magnetic energy. 


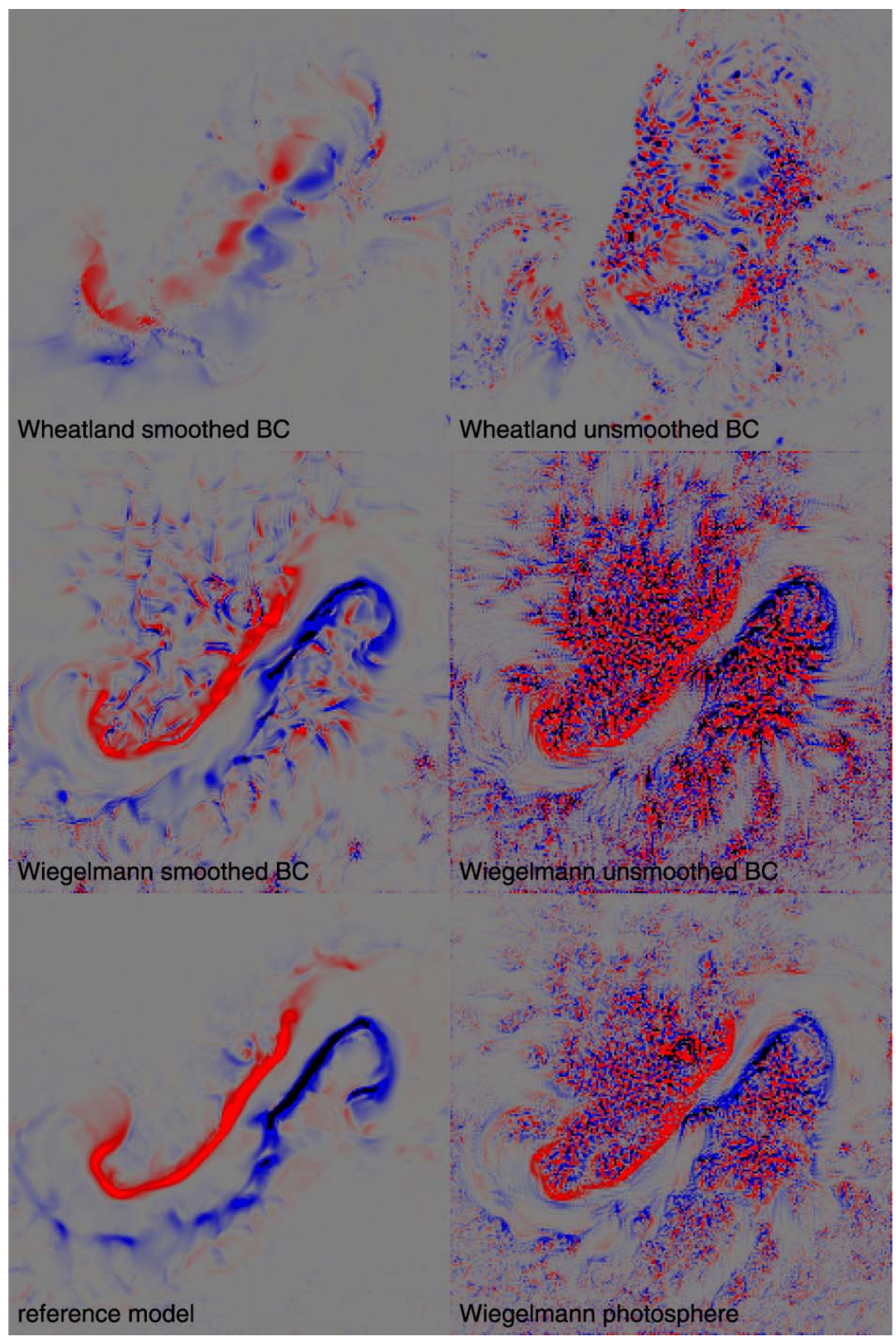

Figure 11 Plots of the vertical current density $J_{z}$ at height $z_{4}\left(0.0004 R_{\odot}\right.$ above the photosphere), for the four photospheric test cases using preprocessed magnetograms (top two rows), as well as for the reference model and for Wiegelmann's solution without preprocessing (bottom row). Smoothing the input vector magnetogram during the preprocessing removes high-frequency currents, which otherwise propagate upward into the corona. 


\section{Discussion and Conclusions}

We have applied a variety of NLFFF extrapolation algorithms, including optimization, magneto-frictional, and Grad-Rubin-like codes, to a solar-like reference model. The van Ballegooijen reference model is much more solar-like than the Low and Lou (1990) models used in Paper I and represents a more realistic test of the NLFFF algorithms. The model includes realistic photospheric Lorentz forces and a complex field including a flux rope.

We find that the optimization method implemented by Wiegelmann is the most successful at modeling the field when the more force-free chromospheric boundary is used as input, though all the codes perform well for the chromospheric test case. However, although all the algorithms were able to qualitatively recover the flux rope from the chromospheric boundary data, none were able to completely recover the field connectivity. Hence, the codes are able to recover the gross character of the field, but are not able to recover all of the fine details. Depending on the application for which the codes are used, these details may or may not be important, but researchers should be cautious about over-interpreting the results from the extrapolations. For example, the details of the connectivity may be important in determining the stability of the flux bundle or in determining the relative magnetic helicity.

The NLFFF algorithms were also confronted with the forced photospheric lower boundary as input. This is an important test of the algorithms, since the forced photospheric vector magnetogram is often the only measurement available, even though it is inconsistent with the force-free assumption of the extrapolation codes. The results are not encouraging. The combination of forces and small spatial scale structure that are present in the photospheric boundary are important and do affect the extrapolations significantly; none of the codes were able to recover the small-scale details of the flux rope when using the photospheric boundary data. Though there is at least a hint of the presence of the flux rope in most of the results, the metrics employed here show that the ability to reconstruct the field from forced boundary data is seriously compromised. In particular, the algorithms were not able to reproduce the energy contained in the field or the field connectivity. The extrapolation codes return a solution which is overall less force-free than the model field, indicating that many details of the model are lost.

The preprocessing of Wiegelmann, Inhester, and Sakurai (2006) removes the net force and torque from the photospheric boundary and gives an improved input to the extrapolation algorithms. When the photospheric boundary is preprocessed, the metrics indicate that the extrapolated field is closer to the model field than in the unpreprocessed photospheric case, at least in the layers above the equivalent of the solar chromosphere in the reference field. In particular, the energy in the field is significantly higher, though the free energy in the photosphere is still not well recovered. When preprocessing the field, it is important to hold the vertical component of the field nearly fixed, though some smoothing is beneficial. If the vertical field is allowed to vary significantly, flux is lost and the energy content of the field is adversely affected, possibly dramatically. The preprocessed photospheric boundary also much improves the estimate of the total energy from the virial theorem for the layers above the chromosphere since the preprocessed boundary is consistent with the assumptions implicit in the virial theorem. Thus, preprocessing is a promising technique and should continue to be developed.

There are residual forces in the model even well above the photosphere. These forces have an impact on the calculation of the total magnetic energy from the virial theorem and are the likely cause of the inability of the codes to correctly recover all of the details of the field connectivity from the chromospheric boundary data. To the extent that such forces are present in the solar corona, the NLFFF codes will similarly have difficulty with the details 
of the real coronal field. However, the extent to which such forces exist on the Sun is not well known. In the next paper in this series, we plan to apply the NLFFF codes to real solar data and use observations of coronal loops from TRACE (Handy et al., 1999) to determine how well the codes perform. These tests may also resolve the question of how important Lorentz forces are in the solar corona and how they impact the NLFFF extrapolation codes.

We re-emphasize here, as concluded in Paper I, that implementation of the boundaries is important in the extrapolation codes. Flux outside the field-of-view can affect the results. In the tests used here, the field-of-view was considerably larger than the volume used in the metrics in order to mitigate this problem. When the codes are applied to solar data, it is important to properly treat the boundaries by using either a very large field-of-view (with spherical rather than Cartesian geometry) or by specifying the side walls of the extrapolation in a way that accounts for flux which is outside of the extrapolation box. We suggest that a global Potential Field Source Surface (PFSS) model (e.g., Riley et al., 2006) might be a good way to set the side walls of the extrapolation to account for magnetic flux that is not included in the box when a full-sphere nonlinear force-free code is prohibitive.

We conclude that nonlinear force-free field modeling approximates the coronal field configuration best if the model is based on a nearly force-free lower boundary, such as the chromosphere might provide in the case of real solar data. The most successful field models in that case have vector metrics approaching the ideal value. These solutions reproduce the appearance of the field lines, the field's general topology, and the value of the free magnetic energy. We thereby demonstrated that the NLFFF tools and methods can rather successfully model fields with a structure and complexity like what we expect to find in solar data, even in the case where the model field was not strictly force-free but contained parameterized buoyancy forces and some residual forces throughout the modeled volume. The residual forces in the model did not seriously impact the extrapolations from the chromospheric boundary, except where details of the field connectivity are concerned, which may be important to certain problems like reconnection geometries. Although this is an important step in demonstrating that NLFFF modeling can be successful under solar conditions, extensive testing based on solar observations will be required as an extension of this study.

When NLFFF modeling is based on a non-force-free boundary, such as for photospheric vector-field data, our conclusion needs to be more conditional. We find that the use of a boundary condition that includes a parameterized buoyancy force yields models that do not satisfactorily reproduce key features of the field morphology, including the flux rope, though the quasi-potential overlying field is better reproduced. The vector metrics are substantially reduced compared to the case for a force-free boundary condition.

Of particular concern for the test case in which a forced photospheric boundary was used, is our finding that the magnetic free energy is not well reproduced. The Wiegelmann solution, for example, finds that the energy in the field is less than that in a potential field. Smoothing the field in the preprocessing discussed in Section 7.3 leads to a significantly improved free-energy estimate, but even that may remain significantly below the true value, depending on the physical interpretation of the preprocessed field. Further study is needed to establish whether the use of a photospheric, non-force-free vector field leads to systematic underestimates of the free energy, and whether it is possible to improve the model field by, for example, incorporating information on the chromospheric field or on the coronal configuration as seen in projection against the disk.

For forced solar boundaries, it is likely that the NLFFF codes will need preconditioned input. Preprocessing dramatically improves the situation but, in cases when a very precise extrapolation is required, it may ultimately be necessary to apply an MHD solution to a time series of vector magnetograms at the lowest heights in order to extrapolate through 
the thin, forced layer before starting a NLFFF extrapolation. Alternatively, a magnetostatic extrapolation might be used to self-consistently compute the extrapolated magnetic field and plasma pressure (Wiegelmann and Neukirch, 2006). In any event, preconditioning of the forced photospheric boundary will require considerable further research.

Acknowledgements This work was supported in part by Independent Research And Development funding of the Lockheed Martin Advanced Technology Center. The work of T. Wiegelmann was supported by DLR-grant 50 OC 0007. The work of G. Barnes and T. Metcalf was supported in part by the National Science Foundation under grant ATM-0454610 and in part by LMATC/IRAD. M.S. Wheatland acknowledges the support, up to June 2006, of an Australian Research Council QEII Fellowship. G. Valori was supported by DFG-grant HO 1424/9-1. The work of J. M. McTiernan at UCB is supported by NASA's Sun-Earth Connection Theory Program, under NNG05G144G-04/08, and NSF's SHINE program, under ATM-0551084-04/10. The authors thank Hugh Hudson, Bernhard Kliem, Yang Liu, Zoran Mikic, Stéphane Régnier, Viacheslav Titov, and Brian Welsch for discussions during the workshop.

\section{References}

Aly, J.J.: 1984, Astrophys. J. 283, 349.

Aly, J.J.: 1989, Solar Phys. 120, 19.

Barnes, G., Leka, K.D., Wheatland, M.S.: 2006, Astrophys. J. 641, 1188.

Chandrasekhar, S.: 1961, Hydrodynamic and Hydromagnetic Stability, Clarendon Press, Oxford.

Chandrasekhar, S., Fermi, E.: 1953, Astrophys. J. 118, 116.

Chodura, R., Schlüter, A.: 1981, J. Comput. Phys. 41, 68.

Gary, G.A.: 1989, Astrophys. J. Suppl. 69, 323.

Gary, G.A., Hagyard, M.J.: 1990, Solar Phys. 126, 21.

Grad, H., Rubin, H.: 1958, Proc. 2nd Int. Conf. Peac. Uses At. Energy 31, 190.

Handy, B.N., Acton, L.W., Kankelborg, C.C., Wolfson, C.J., Akin, D.J., Bruner, M.E., et al.: 1999, Solar Phys. 187, 229.

Hoeksema, J.T.: 1991, Adv. Space Res. 11, 15.

Low, B.C.: 1984. In: M.J. Hagyard (ed.) Measurements of Solar Vector Magnetic Fields, NASA CP 2374, 49.

Low, B.C., Lou, Y.Q.: 1990, Astrophys. J. 352, 343.

Metcalf, T.R., Jiao, L., McClymont, A.N., Canfield, R.C., Uitenbroek, H.: 1995, Astrophys. J. 439, 474.

Metcalf, T.R., Leka, K.D., Mickey, D.L.: 2005, Astrophys. J. 623, L53.

Molodenskii, M.M.: 1969, Sov. Astron. AJ 12, 585.

Priest, E.R.: 1984, Solar Magneto-Hydrodynamics, Reidel, Dordrecht.

Priest, E.R., Démoulin, P.: 1995, J. Geophys. Res. 100(A12), 23443.

Riley, P., Linker, J.A., Mikić, Z., Lionello, R., Ledvina, S.A., Luhmann, J.G.: 2006, Astrophys. J. $653,1510$.

Sakurai, T.: 1979, Publ. Astron. Soc. Japan 31, 209.

Sakurai, T.: 1981, Solar Phys. 69, 343.

Scherrer, P.H., Bogart, R.S., Bush, R.I., Hoeksema, J.T., Kosovichev, A.G., Schou, J., et al.: 1995, Solar Phys. 162, 129.

Schrijver, C.J., DeRosa, M.L.: 2003, Solar Phys. 212, 165.

Schrijver, C.J., DeRosa, M.L., Metcalf, T.R., Liu, Y., McTiernan, J., Régnier, S., et al.: 2006, Solar Phys. 235, 161 .

Valori, G., Kliem, B., Fuhrmann, M.: 2007, Solar Phys. 245, 263.

van Ballegooijen, A.A.: 2004, Astrophys. J. 612, 519.

van Ballegooijen, A.A., DeLuca, E.E., Squires, K., Mackay, D.H.: 2007, J. Atmos. Solar-Terr. Phys. 69, 24.

van Ballegooijen, A.A., Priest, E.R., Mackay, D.H.: 2000, Astrophys. J. 539, 983.

Wang, Y.-M., Sheeley, N.R.: 1990, Astrophys. J. 365, 372.

Wheatland, M.S.: 2004, Solar Phys. 222, 247.

Wheatland, M.S.: 2006, Solar Phys. 238, 29.

Wheatland, M.S., Sturrock, P.A., Roumeliotis, G.: 2000, Astrophys. J. 540, 1150.

Wiegelmann, T.: 2004, Solar Phys. 219, 87.

Wiegelmann, T.: 2007, Solar Phys. 240, 227.

Wiegelmann, T., Neukirch, T.: 2006, Astron. Astrophys. 457, 1053.

Wiegelmann, T., Inhester, B., Sakurai, T.: 2006, Solar Phys. 233, 215.

Wiegelmann, T., Thalmann, J.K., Schrijver, C.J., DeRosa, M.L., Metcalf, T.R.: 2008, Solar Phys. in press.

Yang, W.H., Sturrock, P.A., Antiochos, S.K.: 1986, Astrophys. J. 309, 383. 